\title{
On the origin of the X-ray emission from Herbig Ae/Be stars ${ }^{\star}$
}

\author{
B. Stelzer ${ }^{1}$, G. Micela ${ }^{1}$, K. Hamaguchi ${ }^{2}$, and J. H. M. M. Schmitt ${ }^{3}$ \\ 1 INAF - Osservatorio Astronomico di Palermo, Piazza del Parlamento 1, 90134 Palermo, Italy \\ e-mail: stelzer@astropa.unipa.it \\ 2 Exploration of the Universe Division, NASA Goddard Space Flight Center, Greenbelt, MD 20771, USA \\ 3 Hamburger Sternwarte, Gojenbergsweg 12, 21029 Hamburg, Germany
}

Received 10 February 2006 / Accepted 18 May 2006

\section{ABSTRACT}

\begin{abstract}
Context. Herbig Ae/Be stars are fully radiative and not expected to support dynamo action analogous to their convective lower-mass counterparts, the T Tauri stars. Alternative X-ray production mechanisms, related to stellar winds or star-disk magnetospheres have been proposed, but their X-ray emission has remained a mystery.

Aims. A study of Herbig Ae/Be stars' global X-ray properties (such as detection rate, luminosity, temperature, variability), helps to constrain the emission mechanism by comparison to other types of stars, e.g. similar-age but lower-mass $\mathrm{T}$ Tauri stars, similar-mass but more evolved main-sequence A- and B-type stars, and with respect to model predictions.

Methods. We performed a systematic search for Chandra archival observations of Herbig Ae/Be stars. The superior spatial resolution of this satellite with respect to previous X-ray instrumentation has allowed us to also examine the possible role of late-type companions in generating the observed X-rays.

Results. In the total sample of 17 Herbig Ae/Be stars, 8 are resolved from X-ray emitting faint companions or other unrelated X-ray bright objects within $10^{\prime \prime}$. The detection fraction of Herbig Ae/Be stars is $76 \%$, but decreases to $35 \%$ if all emission is attributed to further known and unresolved companions. The spectral analysis confirms the high X-ray temperatures $(\sim 20 \mathrm{MK})$ and large range of fractional X-ray luminosities $\left(\log L_{\mathrm{x}} / L_{*}\right)$ of this class derived from earlier studies of individual objects.

Conclusions. Radiative winds are ruled out as an emission mechanism on the basis of the high temperatures. The X-ray properties of Herbig Ae/Be stars are not vastly different from those of their late-type companion stars (if such are known), nor from other young late-type stars used for comparison. Therefore, either a similar kind of process takes place in both classes of objects, or there must be as yet undiscovered companion stars.
\end{abstract}

Key words. X-rays: stars - stars: early-type - stars: pre-main sequence - stars: activity - binaries: general

\section{Introduction}

Herbig $\mathrm{Ae} / \mathrm{Be}(\mathrm{HAeBe})$ stars are intermediate-mass stars on the pre-main sequence (pre-MS). With spectral types A to F they represent high-mass counterparts to the T Tauri stars (TTS). Herbig et al. (1960) first introduced HAeBe stars as a class. There are no clear criteria for the definition of this group. One of their major distinctions is strong $\mathrm{H} \alpha$ emission, but according to some definitions an infrared (IR) excess from dusty circumstellar material is considered sufficient to attribute a star to the HAeBe class (see Waters \& Waelkens 1998). In this sense $\mathrm{HAeBe}$ stars are closely related to the debris disk systems such as $\beta$ Pic and Vega, which are probably more evolved. The most comprehensive catalog of HAeBe stars has been compiled by The et al. (1994), including both well-established members of the class and objects that follow a more loose definition, such as emission line stars of spectral type F, non-emission line IR excess stars, and stars that are not found in young associations but appear isolated.

The first systematic studies of X-ray emission from HAeBe stars were presented by Zinnecker \& Preibisch (1994) and by Damiani et al. (1994) using data obtained by the ROSAT and Einstein missions. About 50\% and 30\% of the observed

* Appendix A and Table 2 are only available in electronic form at http://www . edpsciences.org targets were detected, respectively. An extensive study using ASCA and ROSAT data including spectral analysis was published by Hamaguchi et al. (2005), and Skinner et al. (2004) discussed a small sample with emphasis on an XMM-Newton observation of HD 104237.

The X-ray emission from lower-mass pre-MS stars (TTS) arises from magnetically heated and confined coronal structures, possibly with a (small) contribution from accretion. However, HAeBe stars are on radiative tracks, and therefore not expected to drive dynamos able to produce the complex field structure that characterizes stars with convective envelopes. For similar reasons, intermediate-mass stars on the MS are believed to be X-ray dark (Schmitt et al. 1985). In contrast to the A- and B-type stars on the MS the HAeBe stars may possess a shear dynamo that is supplied by the rotational energy of the star. This mechanism was shown to sustain magnetic fields in the initial phase (a few Myrs) of the life of an intermediate-mass star (Tout \& Pringle 1995). Furthermore, HAeBe stars may be young enough to have maintained their primordial fields. However, these fields should be well-ordered and their efficiency in generating X-rays is unclear. Direct measurements of magnetic fields on HAeBe stars are difficult because of the presence of circumstellar matter, the large number of emission lines, and their generally fast rotation. A few marginal detections were reported by Donati et al. (1997), Hubrig et al. (2004), and Wade et al. (2005). In the last study, 
5 of $50 \mathrm{HAeBe}$ stars observed with spectropolarimetric methods showed magnetic signatures in the Stokes $V$ profile, indicating longitudinal fields of a few hundred Gauss at most.

The detection of non-thermal radio emission would be evidence for the presence of magnetic fields, but most of the radio detections among the $\mathrm{HAeBe}$ stars seem to be thermal sources, and were interpreted as being due to a weak stellar wind (Skinner et al. 1993). Further evidence for winds comes from forbidden line emission (Corcoran \& Ray 1997). Winds can also explain the various observed shapes of the $\mathrm{H} \alpha$ emission profile in HAeBe stars (Böhm \& Catala 1995). Instabilities in the wind could give rise to shocks that heat the plasma to X-ray emitting temperatures, in analogy to the case of OB stars. The early X-ray studies of HAeBe stars with Einstein and ROSAT (Damiani et al. 1994; Zinnecker \& Preibisch 1994) came up with correlations between X-ray luminosity and kinetic wind energy $\left(\dot{M} v_{\infty}^{2}\right)$ as well as wind loss rate $(\dot{M})$. However, subsequent spectral studies with $A S C A$ showed X-ray temperatures in excess of $1 \mathrm{keV}$ (Hamaguchi et al. 2005). The winds of HAeBe stars are weaker than those of hot stars, and their terminal speed is probably not sufficient to produce these temperatures (MacFarlane \& Cassinelli 1989). Magnetic fields - if present - may influence the wind geometry and dynamics by channeling the wind as in the MCWS (=Magnetically Confined Wind Shock) model. This scenario was originally developed by Babel \& Montmerle (1997a) to explain the X-ray emission from the Ap star IQ Aur, but was also applied to the O star $\Theta$ Ori C (Babel \& Montmerle 1997b). The same mechanism could be the cause for the strong and variable X-ray emission of some hot stars in Orion (Stelzer et al. 2005). Its application to HAeBe stars depends on whether wind velocities and X-ray temperatures can be reconciled, and seems to be dubious according to previous results.

Some HAeBe stars are still in the accretion phase. The possible role of accretion for the generation of X-rays in low-mass pre-MS stars has recently gained support, most importantly from the high density and soft spectrum of the emitting plasma derived from high-resolution X-ray spectroscopy (Kastner et al. 2002; Stelzer \& Schmitt 2004; Schmitt et al. 2005). Equivalent studies for HAeBe stars are absent from the literature. A possible link between X-ray activity of HAeBe stars and the presence of outflows, typical for stars in the accretion phase, was suggested by Hamaguchi et al. (2005).

Despite the abundant speculations about the possible mechanism, a convincing and unique explanation for the X-ray emission of HAeBe stars has not been identified. A further hypothesis is that of unknown/unresolved T Tauri like companions that would be responsible for the X-ray emission. This idea is the most favored explanation for the observed X-rays from more evolved A- and B-type stars on the MS. Stelzer et al. (2003) and Stelzer et al. (2006) have checked the companion hypothesis by resolving a sample of B-type stars with Chandra from their close visual companions. The result was ambiguous because more than half of the B-type stars were detected with Chandra even after being resolved from all known visual companions. However, the X-ray sources associated with the B-type stars do not show significantly different properties from those coincident with their late-type companion stars. This is fully consistent with the idea that the X-rays originate from even closer spectroscopic companions.

Here we apply the same approach to the sample of HAeBe stars observed with Chandra. Chandra is the only satellite that provides a sub-arcsecond spatial resolution in X-rays, which is reasonably close to IR imaging (adaptive optics) observations. This implies that the majority of known visual companions,

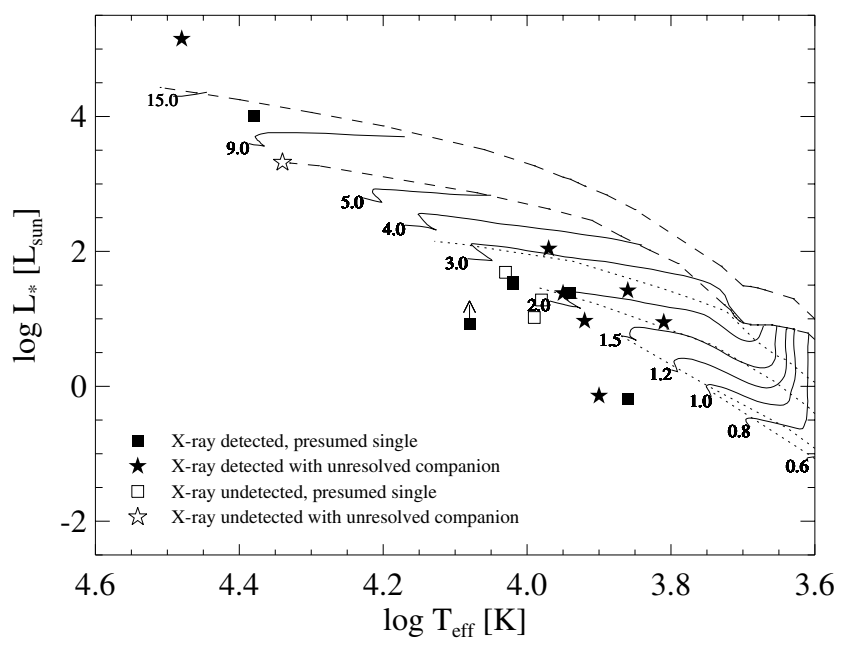

Fig. 1. HR diagram for $\mathrm{HAeBe}$ stars observed with Chandra. Superposed on the data are evolutionary models by Palla \& Stahler (1999): solid lines are tracks labeled by the corresponding mass in solar units, dotted lines are 1,5, and $30 \mathrm{Myr}$ isochrones and dashed lines are the birthlines for accretion rates of $\dot{M}=10^{-4} M_{\odot} / \mathrm{yr}$ and $10^{-5} M_{\odot} / \mathrm{yr}$, respectively.

those at separations larger than $\sim 1^{\prime \prime}$, can be resolved for the first time in X-rays.

Our knowledge of the multiplicity of HAeBe stars is rather incomplete. A few studies have focused on a search for close visual binaries (e.g. Li et al. 1994; Pirzkal et al. 1997; Leinert et al. 1997; Smith et al. 2005). Monte Carlo simulations by Pirzkal et al. (1997) have suggested that almost all HAeBe stars have companions within a completeness limit of $K<10.5 \mathrm{mag}$ for separations of $0.4 \ldots 8^{\prime \prime}$. Corporon \& Lagrange (1999) performed a spectroscopic survey for binaries among HAeBe stars, and discovered 13 binaries by means of radial velocity variations or the detection of lithium absorption.

This paper is structured as follows. In Sect. 2 we introduce the targets observed with Chandra. The observations and the data analysis are described in Sect. 3. Section 4 summarizes the results from the source detection, the temporal and the spectral analyses. In the discussion (Sect. 5) we examine the nature of the X-ray emitters, the global X-ray properties of HAeBe stars, the $L_{\mathrm{x}} / L_{\text {bol }}$ relation, and correlations between $\mathrm{X}$-ray emission and other characteristic stellar parameters. Section 6 presents the conclusions. Information on individual stars is found in Appendix A.

\section{The sample}

We have searched the Chandra archive for observations of HAeBe stars. Positional cross-correlation with the catalogs by The et al. (1994), van den Ancker et al. (1997), and van den Ancker et al. (1998) identified 17 objects. Their position in the HR diagram is shown in Fig. 1. The stellar parameters were compiled from the literature and are given together with the references in Table 1. Two stars, V892 Tau and R CrA, are located well below the MS in Fig. 1, probably indicating a serious problem in the calculation of $L_{*}$ due to the strong and poorly constrained extinction; see also Sect. 5.3.

In Fig. 1 and in Table 1 we anticipate the results from the Chandra source detection process discussed further in Sect 3.1. The last three columns of Table 1 summarize the X-ray detection status of all HAeBe stars, and whether there are known 
Table 1. Stellar parameters for HAeBe stars observed with Chandra (left side) and parameters relevant for the interpretation of the X-ray emission (right side). The references in Col. 5 refer to Cols. 2-4, separated by commas. The flags in Cols. 8-10 stand for X-ray detection ("X"), presence of unresolved visual companion ("VB") and spectroscopic companion ("SB").

\begin{tabular}{|c|c|c|c|c|c|c|c|c|c|}
\hline Designation & $\begin{array}{r}A_{\mathrm{V}} \\
{[\mathrm{mag}]}\end{array}$ & $\begin{array}{r}\log T_{\text {eff }} \\
{[\mathrm{K}]}\end{array}$ & $\log \left(L_{\text {bol }} / L_{\odot}\right)$ & Ref. & $\begin{array}{r}v \sin i \\
{[\mathrm{~km} / \mathrm{s}]}\end{array}$ & Ref. & $\mathrm{X}$ & VB & SB \\
\hline $\mathrm{BD}+30^{\circ} 549$ & 1.89 & 4.08 & $>0.93$ & $(1,1,1)$ & & & $\sqrt{ }$ & & \\
\hline V892 Tau & $3.9 \ldots 12.5$ & 3.90 & -0.14 & $(7,8,8)$ & & & $\sqrt{ }$ & $\sqrt{ }$ & \\
\hline V380 Ori & $1.43 \ldots 1.7$ & 3.97 & 2.04 & $(1 \ldots 2,1,3)$ & 200 & (4) & $\sqrt{ }$ & $\sqrt{ }$ & $\sqrt{ }$ \\
\hline HD 147889 & 3.32 & 4.34 & 3.32 & $(6,6,6)$ & $30 . .70$ & (4) & - & & $\sqrt{ }$ \\
\hline V590 Mon & $0.6 \ldots 1.3$ & 3.99 & 1.02 & $(2 \ldots 5,2,3)$ & & & - & & \\
\hline $\mathrm{ZCMa}$ & $2.4 \ldots 4.46$ & $3.8 \ldots 4.48$ & $>1.72 \ldots .5 .15$ & $(7 \ldots 8,1 \ldots 8,1 \ldots 8)$ & $<130$ & (1) & $\sqrt{ }$ & $\sqrt{ }$ & \\
\hline HD 97300 & 1.33 & 4.02 & 1.54 & $(6,6,6)$ & & & $\sqrt{ }$ & & \\
\hline HD 100546 & $0.2 \ldots 1.0$ & 4.02 & 1.51 & $(7,1,1)$ & 250,55 & $(1,9)$ & $\sqrt{ }$ & & \\
\hline HD 104237 & $0.3 \ldots 1.3$ & 3.86 & 1.42 & $(7,8,8)$ & 10 & (9) & $\sqrt{ }$ & & $\sqrt{ }$ \\
\hline HD 141569 & 0.47 & 3.98 & 1.28 & $(1,8,8)$ & 236,200 & $(1,4)$ & - & & \\
\hline HD 150193 & 1.61 & 3.95 & 1.38 & $(1,8,8)$ & 100 & (8) & $\sqrt{ }$ & & $\sqrt{ }$ \\
\hline HD 152404 & 0.62 & 3.81 & $0.88 \ldots 0.95$ & $(8,1,1 \ldots 8)$ & 19 & (1) & $\sqrt{ }$ & & $\sqrt{ }$ \\
\hline HD 163296 & $0.09 \ldots 0.3$ & 3.94 & $1.38 \ldots 1.48$ & $(8 \ldots 7,8,8 \ldots 1)$ & 120 & (10) & $\sqrt{ }$ & & \\
\hline MWC 297 & $2.9 \ldots 8.3$ & 4.38 & 4.01 & $(7,8,8)$ & & & $\sqrt{ }$ & & \\
\hline HD 176386 & 0.62 & 4.03 & 1.69 & $(6,6,6)$ & & & - & & \\
\hline TY CrA & $1.0 \ldots 3.1$ & 3.92 & 0.97 & $(7,2,2)$ & 10,88 & $(4,2)$ & $\sqrt{ }$ & $\sqrt{ }$ & $\sqrt{ }$ \\
\hline $\mathrm{R} \mathrm{CrA}$ & $0.9 \ldots 1.9$ & $3.86 \ldots 3.81$ & $-0.19 \ldots-0.38$ & $(7,8 \ldots 2,8 \ldots 2)$ & & & $\sqrt{ }$ & & \\
\hline
\end{tabular}

(1) van den Ancker et al. (1998); (2) Hillenbrand et al. (1992); (3) Corcoran \& Ray (1998); (4) Corporon \& Lagrange (1999); (5) Hernandez et al. (2004); (6) van den Ancker et al. (1997); (4) Haffner \& Meyer (1995); (7) Elia et al. (2004); (8) Acke \& van den Ancker (2004); (9) Acke \& Waelkens (2004b); (10) Finkenzeller (1985).

companions that remained unresolved with Chandra. In Fig. 1 binaries that remain unresolved with Chandra are marked with different plotting symbols. Information on companions was collected from the literature. We focus on close systems that could not be resolved with previous X-ray satellites. About half of these companions are separable from the HAeBe stars with Chandra (those with separation $\geq 1^{\prime \prime}$ ). Physical companionship has not been established for most of these objects. Confirmation that they are true companions, and not just chance projections, requires observations of their proper motion and/or spectra.

For many of the targets the Chandra data have already been discussed in some detail in the literature, but no homogeneous analysis of these observations has been presented so far. Furthermore, we add some updates and corrections with respect to previous publications. The list of all targets is summarized in Table 2 that gives the designation, position, distance, and spectral type of the HAeBe star, as well as separation, position angle, an identifier flag for all known companions, and the respective reference. Some basic information about the Chandra observations is given at the end of Table 2, where the reference column refers to previous citations of the Chandra data.

The data were obtained within various unrelated observing programs. Therefore, the sample observed with Chandra may not be representative of the total population of HAeBe stars. As can be seen from Fig. 1 the stars are distributed unevenly across the HR diagram. The observations are also not homogeneous in terms of sensitivity limit, because of the different distances and exposure times. Most important for our study is a possible bias towards known X-ray emitters.

\section{Observations and data analysis}

Most targets were observed with the standard imaging array of Chandra's Advanced CCD Imaging Spectrometer (ACIS-I); see Weisskopf et al. (2002) for details on the satellite and its instruments. In some cases the target was placed on the backside illuminated ACIS-S3 chip. The observing instrument is indicated in Col. 11 of Table 2.

Data analysis was carried out using the CIAO software package $^{1}$ version 3.2 in combination with the calibration database (CALDB) version 3.0.0. We started our analysis with the level 1 events file provided by the Chandra X-ray Center (CXC). On all observations processed at the CXC with CALDB version earlier than 2.9 we applied a new gain map and updates on the geometry (focal length, ACIS pixel size and chip positions). For observations processed with a later version of CALDB these modifications had been performed automatically during the pipeline process. In the process of converting the level 1 events file to a level 2 events file for each of the observations we performed the following steps: a correction for the charge transfer inefficiency (CTI) has been applied for data with processing version earlier than 6.12, i.e. for those where the CTI correction was not yet part of the standard pipeline processing at the CXC. We removed the pixel randomization which is automatically applied by the CXC pipeline in order to optimize the spatial resolution. We filtered the events file for event grades (retaining the standard grades $0,2,3,4$, and 6), and applied the standard good time interval file. Events flagged as cosmic rays were not removed in our analysis. In principle, such events can lead to the detection of spurious sources. However, if identified on the position of a bright X-ray source, the flag is often erroneous (as a result of the event pattern used for the identification of cosmic rays). Inspection of our images revealed that a substantial number of events at the positions of our sources carry the cosmic ray flag (typically 3-5\%; see http://cxc.harvard.edu/ ciao/threads/acisdetectafterglow). Removing these events would therefore result in an underestimate of the source count rate.

Since the positional accuracy is particularly important to our observations we also checked the astrometry for any known

1 CIAO is made available by the CXC and can be downloaded from http://cxc.harvard.edu/ciao/download-ciao-reg.html 
systematic aspect offsets using the CIAO aspect calculator ${ }^{2}$. Small offsets are present in all of the presented observations, and the aspect was corrected accordingly by modifying the respective header keywords in the level 2 events file.

\subsection{Source detection}

Source detection was restricted to a $50 \times 50$ pixel wide image $\left(1\right.$ pixel $\left.=0.492^{\prime \prime}\right)$, centered on the position of the primary HAeBe star. Exceptions are HD 104237, where the image center was offset to include all obvious nearby X-ray sources, and R CrA where visual inspection of the image identifies two $\mathrm{X}$-ray emitters at slightly wider separations, and source detection was performed on a $100 \times 100$ pixels large area. Source detection was carried out with the WAVDETECT algorithm (Freeman et al. 2002). This algorithm correlates the data with a Mexican Hat function to search for deviations from the background. The WAVDETECT mechanism is well suited for separating closely spaced point sources. We used wavelet scales between 1 and 8 in steps of $\sqrt{2}$. Two of the targets (HD 100546 and HD 104237) were observed twice, and we merged the observations to obtain a combined image with higher $\mathrm{S} / \mathrm{N}$.

\section{Results}

Figure 2 shows a portion of the ACIS images centered on the HAeBe stars. In general, the scale of the images displayed in this figure corresponds to the area in which source detection was performed. However, in some cases we show a smaller image fraction in order to provide a better view of crowded fields. The photon extraction areas of all detected X-ray sources are overplotted (circles), as well as the position of the primary and the position of the companions (x-shaped symbols). For the case of HD 141569 and HD 150193 our identifications differ from the results presented by Feigelson et al. (2003); some of the erroneous results were also used by Skinner et al. (2004). For a detailed discussion of this issue we refer to Appendices A.10 and A.11.

Source identification was done by measuring the separation of each X-ray source from the optical/IR positions of all known visual components. Then we assigned each X-ray source to the closest of the optical/IR objects. This procedure turned out to be unambiguous except in cases where the separation of the components is below Chandra's spatial resolving power, such that a single X-ray source represents more than one visual component. In the HD 150193 system only one elongated X-ray source is detected, identified with component $\mathrm{B}$, but the image clearly shows an enhancement of photons in the direction of component A. Therefore, we consider HD $150193 \mathrm{~A}$ also as an X-ray source; see the footnote of Table 3 for details.

Table 3 summarizes the identification of all X-ray sources with components of our target systems and their X-ray parameters. We list also parameters for X-ray sources detected in the examined image portion but not known to be associated with the HAeBe targets. Columns 1-5 give the designation of the target, component identifier, X-ray source number, offaxis angle, and offset between X-ray and optical/IR position. For X-ray sources that are not known to be associated with the targets, we give an identifier in Col. 2. Column 6 represents the significance of the detection. The number of counts $N$ (Col. 7) refers to the

\footnotetext{
2 See http://asc.harvard.edu/ciao/threads/ arcsec_correction
}

0.5-8 keV passband. Errors were computed with the Gehrels approximation $\sqrt{N+0.75}+1$ for Poisson distributed data (Gehrels 1986). The irrelevant influence of the background is obvious from Fig. 2. To quantify this, we estimated the background within a squared area of $1^{\prime}$ side length centered on the optical/IR position of the respective star but excluding all detected sources. After scaling to the source extraction area this background is negligibly small $\left(<10^{-5} \mathrm{cps}\right)$, and therefore not considered in the analysis. As a rule, source photons were extracted from a circle centered on the WAVDETECT source position within the radius that includes $90 \%$ of the point-spread-function (PSF) at $1.5 \mathrm{keV}$, depending on the position of the source on the detector. For sources that are not fully resolved we defined smaller nonoverlapping circular photon extraction areas (see Col. 10). To compute upper limits for the undetected components of our target systems we used the method for Poisson-distributed counting data described by Kraft et al. (1991). Columns 8 and 9 of Table 3 show hardness ratios defined as $H R=(H-S) /(H+S)$, where $H$ and $S$ are the number of counts in a hard band and in a soft band, respectively. $H R 1$ is defined using emission in the $0.5-1 \mathrm{keV}(S)$ and the $1-8 \mathrm{keV}(H)$ bands, and $H R 2$ from the $1-2 \mathrm{keV}(S)$ and the $2-8 \mathrm{keV}(H)$ band. Column 10 is the PSF fraction included in the source extraction area. In Cols. 11 and 12 the PSF- and absorption-corrected X-ray luminosity in the $0.5-8 \mathrm{keV}$ band and the ratio of X-ray to bolometric luminosity are given. The $\mathrm{X}$-ray luminosities have been computed with PIMMS ${ }^{3}$ assuming an iso-thermal emitting plasma with $k T=1 \mathrm{keV}$ and an absorbing column density $N_{\mathrm{H}}$ corresponding to the value derived from $A_{\mathrm{V}}$ according to the extinction law of Ryter (1996) $\left(N_{\mathrm{H}}\left[10^{22} \mathrm{~cm}^{-2}\right]=A_{\mathrm{V}}[\mathrm{mag}] \times 2.0 \times 10^{21}\right)$. The bolometric luminosity represents the blackbody radiation from the stellar photosphere, without taking into account excess emission from circumstellar material seen at IR and radio wavelengths; see Table 1 for the stellar parameters of the HAeBe stars. The assumption of an iso-thermal plasma may not be appropriate. However, we show in Sect. 4.2 that the X-ray luminosities derived in this way are in reasonable agreement with the values obtained from the actual X-ray spectrum. Finally, Col. 13 of Table 3 represents the significance of variability according to the KS-test.

\subsection{X-ray lightcurves}

Lightcurves were binned for all detected sources into $400 \mathrm{~s}$ and $800 \mathrm{~s}$ intervals. Obviously, the detection of variability requires both high statistics and a good time resolution, and our choice of the binning is a compromise between these two opposing effects. The probabilities for variability according to the KS-test are summarized in the last column of Table 3. HD 100546 and HD 104237 were observed twice, and the time-series of both data sets were analysed separately, to avoid mixing long and short timescales. The detection of variability (at the $99 \%$ confidence level) in only 5 of the 29 examined time series may be due to the short exposure times of some observations. In Fig. 3 the lightcurves of the variable sources are displayed.

As a further test on variability we applied a method that determines periods of constant signal from a list of photon arrival times, based on a maximum likelihood algorithm under the assumption of Poisson statistics. This technique is termed MLB (maximum likelihood blocking) henceforth, see Wolk et al. (2005) for a more detailed description. The algorithm has two free parameters: the minimum number of counts accepted in a

\footnotetext{
3 The Portable Interactive Multi-Mission Simulator (PIMMS) is accessible at http://asc . harvard. edu/toolkit/pimms . jsp
} 

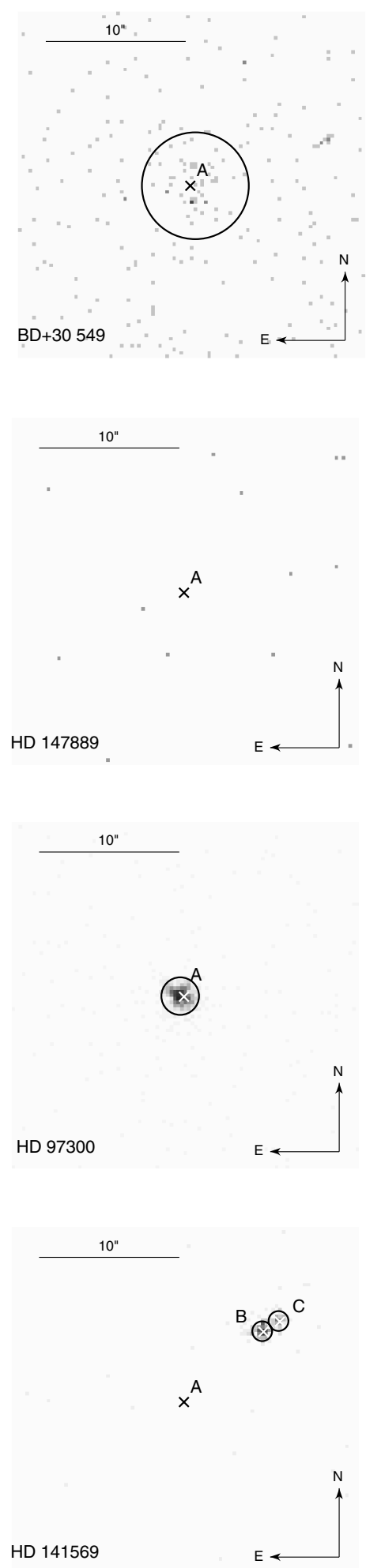

$$
{ }^{B}{ }^{\mathrm{C}}
$$
$x^{\mathrm{A}}$

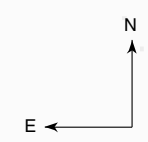

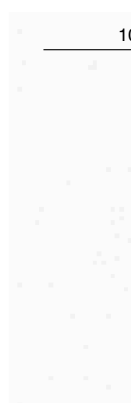

V892 Tau
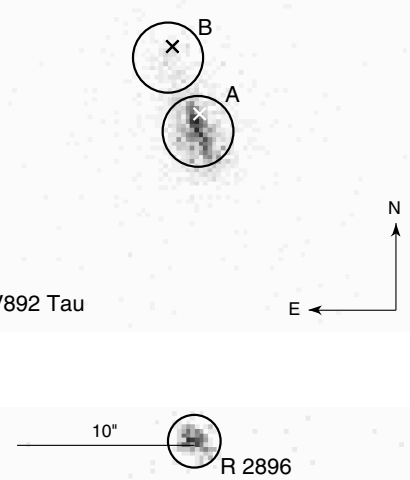

$$
x^{\mathrm{A}}
$$
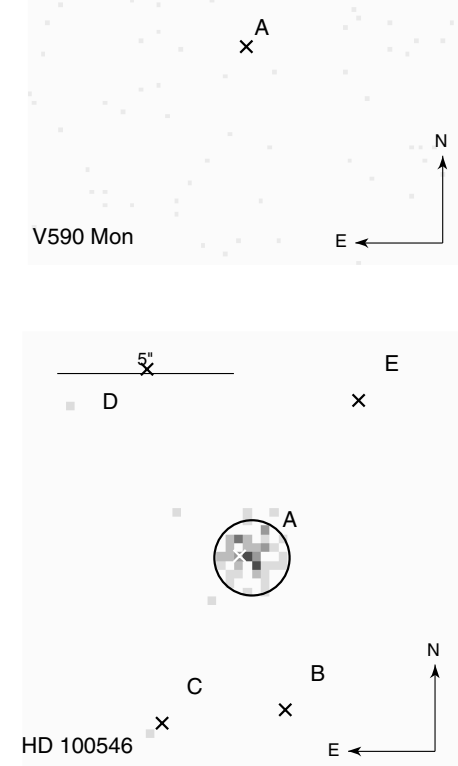

$5^{\prime \prime}$

A

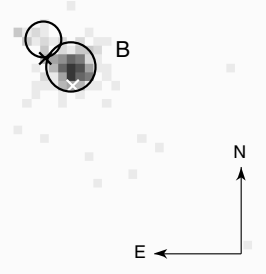

HD 150193
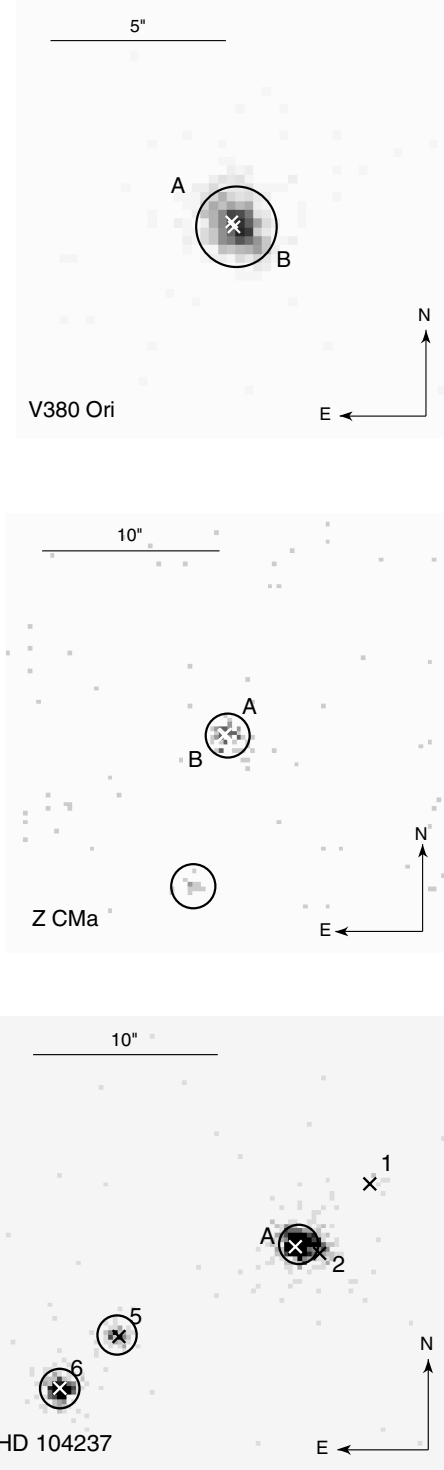

$5^{\prime \prime}$

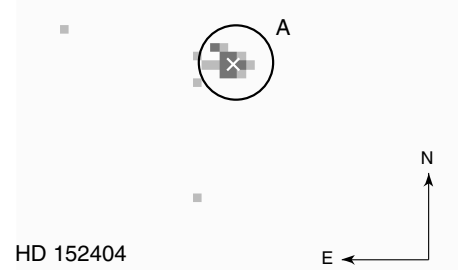

HD 152404

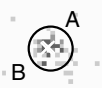

0

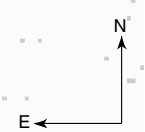

Fig. 2. Chandra ACIS images of HAeBe stars binned to a pixel size of $0.25^{\prime \prime}$. Crosses denote the optical/IR position of the individual components in the multiple system, circles mark the photon extraction areas centered on the position of X-ray sources detected with WAVDETECT.

given segment and the significance, defined as $1-P$, where $P$ is the probability that a constant lightcurve is spuriously segmented due to random fluctuations. We set the significance to $99 \%$, and the minimum number of counts in a segment was 20, except for weak sources, where we allowed as few as a single count to define a segment. The segmented lightcurves of the sources found to be variable with the KS-test are overplotted on the binned curves in Fig. 3. 

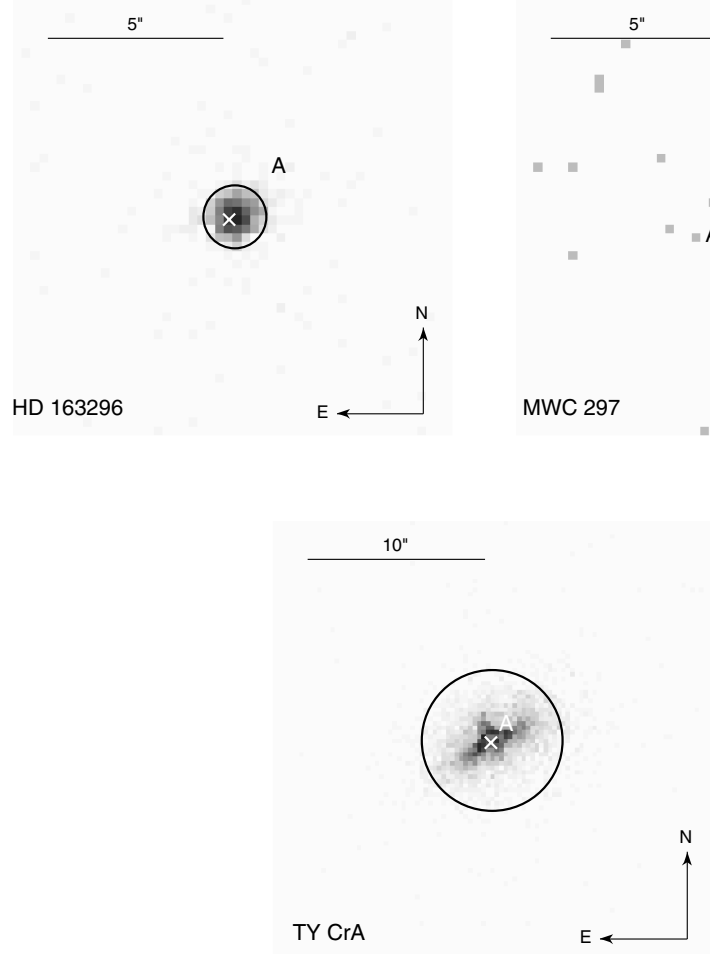
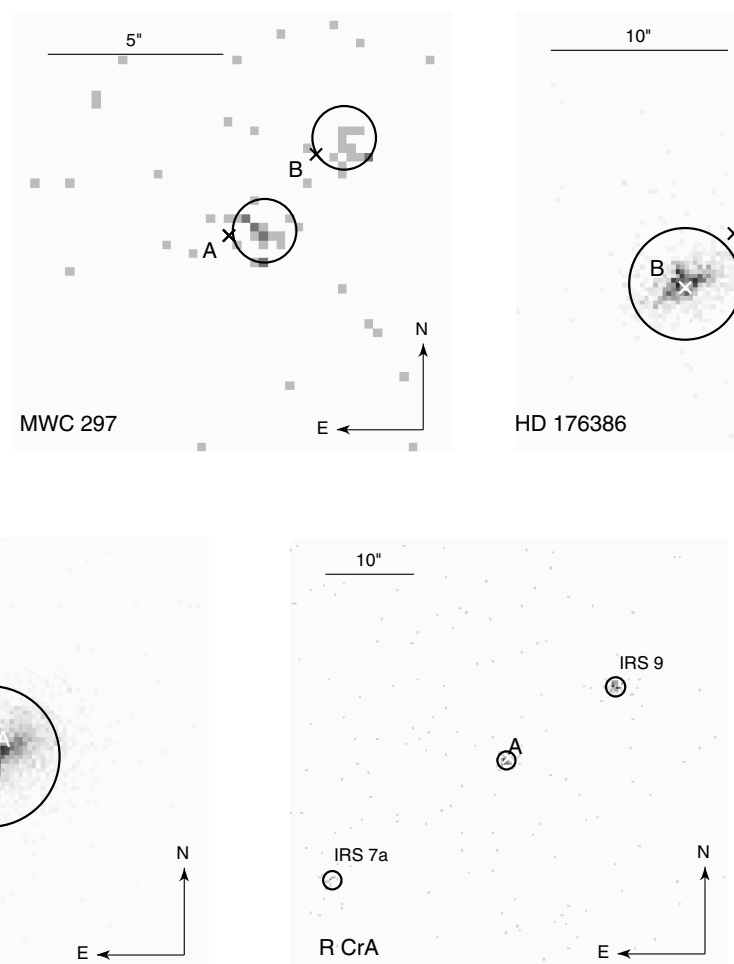

Fig. 2. continued.

We find excellent agreement between the detection of variability with the KS-test and with the MLB method. All stars variable according to the KS-test are also found to be variable with the MLB technique, while all stars non-variable according to the KS-test are described by a single segment in the MLB analysis. With the exception of TY CrA the luminosity is not significantly affected by flaring, and we do not evaluate flare and quiescent data separately.

\subsection{X-ray spectra}

The preparations for the spectral analysis consist of the following steps: we extracted a spectrum, the corresponding detector response matrix that maps pulse heights into energy space, and an auxiliary response file which contains information about the effective area and detector efficiency across the chip as a function of energy. Then we binned each spectrum to a minimum of 5 or more counts per bin starting at $0.5 \mathrm{keV}$. As the background of ACIS is very low ( $<1$ count in the source extraction area) it can be neglected. For several X-ray sources in the sample presented here the number of counts collected is rather small, and the results are discussed only for the 17 sources with at least five degrees of freedom after binning the spectrum and choosing an adequate model. Since the models have at least 2 free parameters (see below), all analysed sources have more than 35 counts. In practice, the lowest $\mathrm{S} / \mathrm{N}$ spectrum $\left(\mathrm{BD}+30^{\circ} 549\right)$ has $>40$ counts. Spectral modelling was performed in the XSPEC environment, version 11.3.0.

We tried four different models on each spectrum: oneand two-temperature thermal models with fixed or freely variable absorption from interstellar and circumstellar material. The thermal components are represented by $\mathrm{APEC}^{4}$ with atomic cross-sections and elemental abundances from Wilms et al. (2000). For the fits with fixed absorption, the column density $N_{\mathrm{H}}$ of the WABS component was set to the values derived from the visual extinction assuming the Ryter (1996) extinction law, and the column density of the companions is assumed to be equal to that of the HAeBe stars, i.e. all components in a given system are considered to be hidden behind the same amount of interstellar plus circumstellar absorbing material. Global metal abundances were fixed to 0.3 times the solar value.

The minimum number of free parameters is two $(k T, E M)$, and the maximum is five $\left(N_{\mathrm{H}}, k T_{1}, k T_{2}, E M_{1}, E M_{2}\right)$. For each model spectral fitting was started on several choices for the initital parameters, and then the fit with the minimum $\chi^{2}$ was adopted. Then we decided for each star individually which of the four models represents best the data. We base the evaluation of the quality of a fit result on the null probability that the spectrum is described by the model. Generally, simpler models are considered first, because an excessive number of free parameters with respect to the data statistics does not yield physical constraints (cf. discussion by Flaccomio et al. 2006). If $P_{\text {Null }}>5 \%$ we accept the 1-T model with free column density $(1 \mathrm{~T})^{5}$. Otherwise,

\footnotetext{
${ }^{4}$ For a description of the Astrophysical Plasma Emission Code (APEC) see Smith et al. (2001).

5 A few exceptions do not satisfy our criterion of $P_{\text {Null }}>5 \%$ in any of the four models. HD 104237-6 is among the fainter sources and shows erratic jumps in the spectrum. For the rather bright X-ray sources corresponding to V380 Ori and HD 163296 the discrepancies between the observed spectrum and the models can probably be attributed to peculiar abundance patterns. Since we are interested in the global properties of HAeBe stars rather than a detailed examination of individual sources, we judge these fits as acceptable after visual inspection.
} 


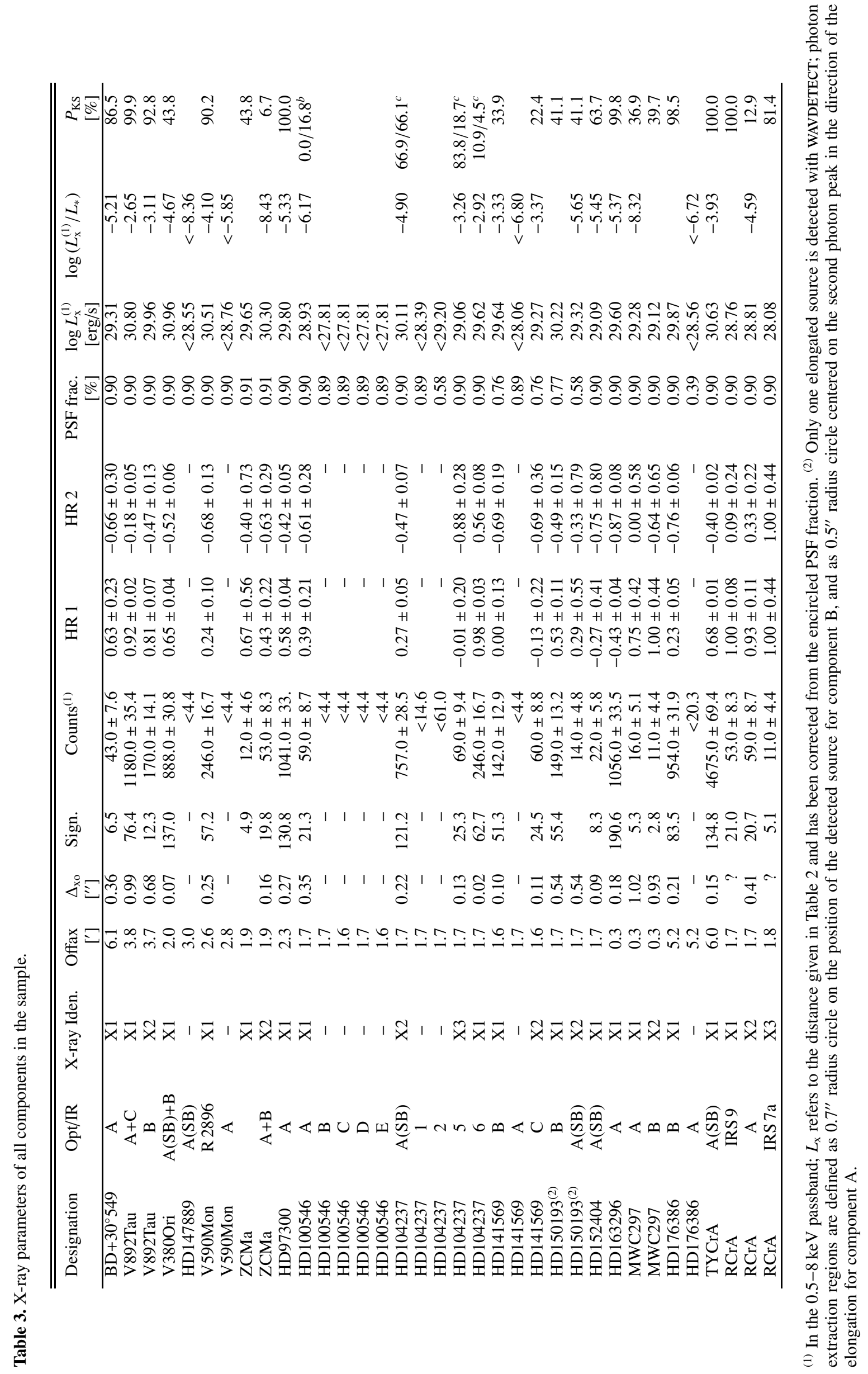



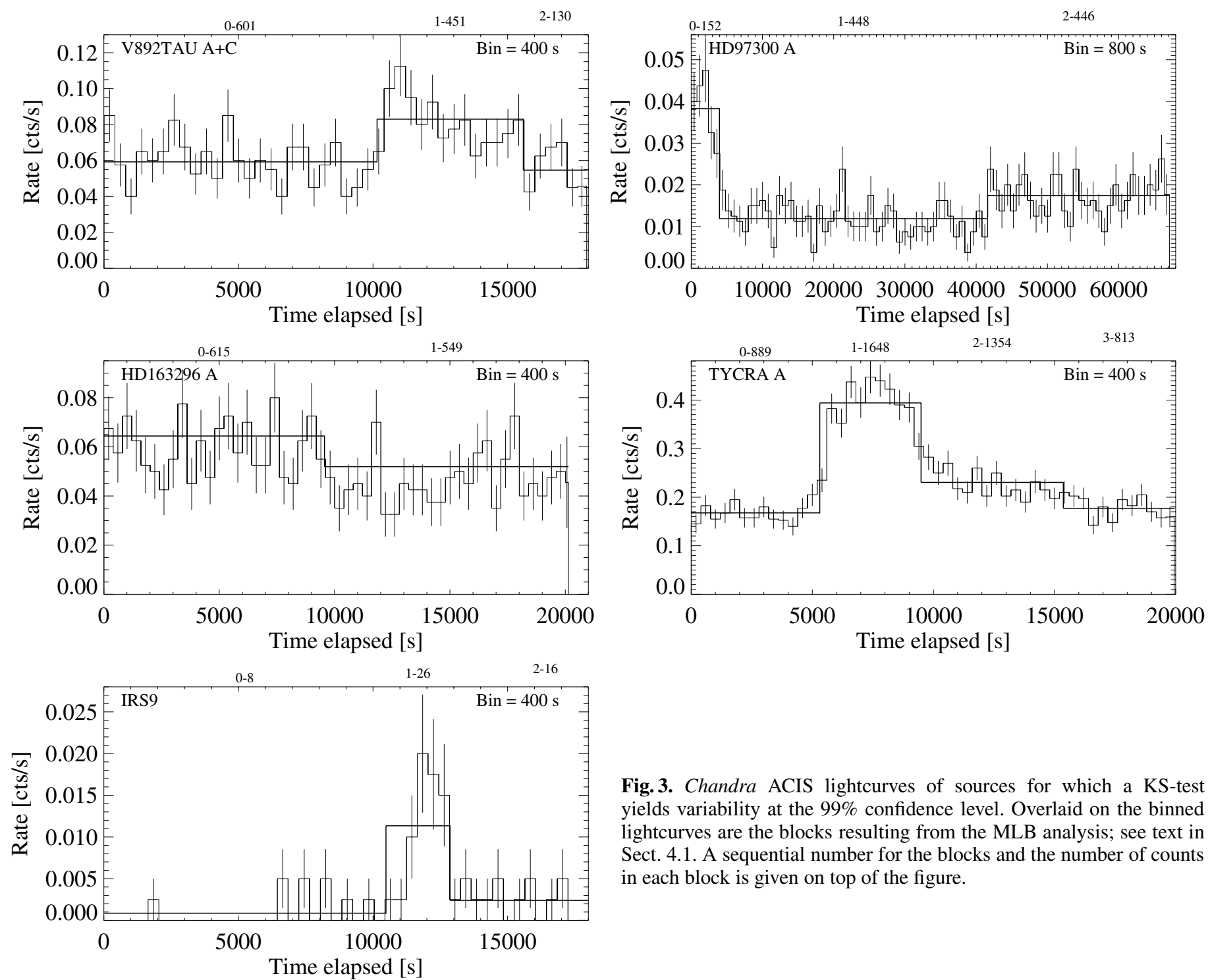

Fig. 3. Chandra ACIS lightcurves of sources for which a KS-test yields variability at the $99 \%$ confidence level. Overlaid on the binned lightcurves are the blocks resulting from the MLB analysis; see text in Sect. 4.1. A sequential number for the blocks and the number of counts in each block is given on top of the figure.

we move on to the 1-T model with fixed $N_{\mathrm{H}}\left(1 \mathrm{~T}_{\mathrm{Av}}\right)$. The preference of $1 \mathrm{~T}$ versus $1 \mathrm{~T}_{\mathrm{Av}}$ despite its fewer degrees of freedom is empirical: The $1 \mathrm{~T}$ model is the one that gives in general better fits than the $1 \mathrm{~T}_{\mathrm{Av}}$ model, probably because $1 \mathrm{~T}_{\mathrm{Av}}$ sometimes ends up in a local minimum of the $\chi^{2}$-space. An example of such a case is $\mathrm{BD}+30^{\circ} 549$, whose best-fit model has too large $N_{\mathrm{H}}$ compared to its $A_{\mathrm{V}}$, and, to compensate for this, has untypically small $k T$ and large $L_{\mathrm{x}}$ (see also Sect. 4.3). The preference of 1T is also motivated by the fact that there are considerable uncertainties in the visual extinction of the targets, such that even under the assumption of the Ryter (1996) extinction law the column density is not well known. If none of the 1-T models is satisfactory, the 2-T model with fixed $N_{\mathrm{H}}$ is chosen $\left(2 \mathrm{~T}_{\mathrm{Av}}\right)$. Finally, the last option is the 2-T model with free absorption (2T).

The parameters derived from spectral fitting are presented in Table 4. Luminosities refer to the $0.5-8.0 \mathrm{keV}$ band after correction for absorption. A comparison of the X-ray luminosities derived from integrating the X-ray spectrum and those calculated from the count rate with PIMMS shows that the values lie within a factor of two of each other, with one exception $\left(\mathrm{BD}+30^{\circ} 549\right)$ discussed below. This proves that our simple estimate with PIMMS yields reliable X-ray luminosities even if the assumed spectral parameters may not precisely reflect the

conditions in the emitting plasma. Throughout the remainder of this paper both luminosities will be used: in the direct comparison of parameters from the X-ray spectrum $L_{\mathrm{X}, \mathrm{SPEC}}$ is used. Otherwise $L_{\mathrm{x}, \text { PIMMS }}$ is preferred because it allows us to consider all targets, including faint sources and non-detections.

\subsection{Peculiar spectral properties}

The majority of stars are characterized by X-ray temperatures of $\sim 1-2.5 \mathrm{keV}$, similar to earlier results from ASCA (Hamaguchi et al. 2005), and moderate extinction. A deviation from this general picture is seen in four sources: the HAeBe stars $\mathrm{BD}+30^{\circ} 549$ and $\mathrm{R} \mathrm{CrA}$, the Class I protostar IRS 9 in the R CrA star forming complex, and the probably accreting T Tauri star HD 104237-6, are strongly absorbed $\left(N_{\mathrm{H}}>10^{22} \mathrm{~cm}^{-2}\right)$. Except for $\mathrm{BD}+30^{\circ} 549$, these stars show also rather high temperatures. All of them are relatively faint, such that the shape of their spectrum cannot be examined in more detail.

For BD $+30^{\circ} 549$ the typical degeneracy of $N_{\mathrm{H}}$ with $k T$ often observed in spectra with low statistics may have led to an incorrect estimate of both parameters (cf. discussion in the previous paragraph). Nevertheless, we retain this result as the best fit because fixing the absorbing column yielded a much poorer $\chi^{2}$ and residuals. 
Table 4. Spectral parameters of X-ray sources associated with HAeBe stars and their known close companions. The "=" sign indicates fits with column densities fixed on the value derived from the optical extinction; see text in Sect. 4.2.

\begin{tabular}{|c|c|c|c|c|c|c|c|c|c|c|}
\hline HD & Opt/IR & X-ray Iden. & $\overline{\chi_{\text {red }}^{2}(\text { dof })}$ & $P_{\text {Null }}$ & $\begin{array}{r}N_{\mathrm{H}} \\
{\left[10^{22} \mathrm{~cm}^{-2}\right]}\end{array}$ & $\begin{array}{r}k T_{1} \\
{[\mathrm{keV}]}\end{array}$ & $\begin{array}{r}k T_{2} \\
{[\mathrm{keV}]}\end{array}$ & $\begin{array}{r}\log E M_{1} \\
{\left[\mathrm{~cm}^{-3}\right]}\end{array}$ & $\begin{array}{r}\log E M_{2} \\
{\left[\mathrm{~cm}^{-3}\right]}\end{array}$ & $\begin{array}{l}\log L_{\mathrm{x}}{ }^{*} \\
{[\operatorname{erg} / \mathrm{s}]}\end{array}$ \\
\hline \multicolumn{11}{|c|}{ HAeBe stars } \\
\hline $\mathrm{BD}+30^{\circ} 549$ & A & $X-1$ & $0.83(5)$ & 0.526 & 1.87 & 0.3 & - & 53.9 & - & 30.4 \\
\hline V892 Tau & $A+C$ & $X-1$ & $0.99(47)$ & 0.493 & 1.36 & 2.0 & - & 53.7 & - & 30.7 \\
\hline V380 Ori & $\mathrm{A}(\mathrm{SB})+\mathrm{B}$ & $X-1$ & $1.62(43)$ & 0.006 & 0.48 & 1.6 & - & 54.4 & - & 31.3 \\
\hline $\mathrm{ZCMa}$ & $A+B$ & $X-2$ & $0.29(7)$ & 0.957 & 0.29 & 1.2 & - & 53.6 & - & 30.5 \\
\hline HD 97300 & A & $X-1$ & $1.13(39)$ & 0.269 & $=0.27$ & 0.8 & 2.5 & 51.9 & 52.8 & 29.9 \\
\hline HD $104237^{a}$ & $\mathrm{~A}(\mathrm{SB})$ & $X-2$ & $1.34(21)$ & 0.138 & $=0.06$ & 0.8 & 4.0 & 53.1 & 53.0 & 30.3 \\
\hline HD $104237^{b}$ & $\mathrm{~A}(\mathrm{SB})$ & $X-1$ & $1.31(17)$ & 0.172 & $=0.06$ & 0.7 & 5.1 & 53.1 & 52.7 & 30.2 \\
\hline HD 163296 & A & $X-1$ & $1.53(33)$ & 0.027 & $=0.05$ & 0.5 & 10.9 & 52.9 & 51.9 & 29.7 \\
\hline TY CrA & $\mathrm{A}(\mathrm{SB})$ & $X-1$ & $1.03(128)$ & 0.383 & 0.49 & 0.8 & 3.2 & 53.5 & 53.7 & 30.9 \\
\hline $\mathrm{R} \mathrm{CrA}$ & A & $X-2$ & $0.72(8)$ & 0.675 & 1.11 & $>2.8$ & - & 51.9 & - & 29.1 \\
\hline \multicolumn{11}{|c|}{ Companions and unrelated objects } \\
\hline V892 Tau & B & $\mathrm{X}-2$ & $0.66(13)$ & 0.801 & 0.76 & 1.7 & - & 52.8 & - & 29.7 \\
\hline $\mathrm{HD} 104237^{a}$ & 6 & $X-1$ & $1.97(11)$ & 0.027 & 3.58 & 2.2 & - & 53.6 & - & 30.7 \\
\hline HD 141569 & B & $X-1$ & $0.91(9)$ & 0.513 & 0.07 & 0.8 & - & 52.9 & - & 29.7 \\
\hline HD 141569 & $\mathrm{C}$ & $X-2$ & $0.47(8)$ & 0.879 & 0.00 & 0.8 & - & 52.4 & - & 29.2 \\
\hline HD 150193 & B & $X-1$ & $1.19(11)$ & 0.288 & 0.15 & 1.5 & - & 53.3 & - & 30.1 \\
\hline HD 176386 & B & $\mathrm{X}-1$ & $1.09(45)$ & 0.318 & $=0.12$ & 1.0 & - & 53.1 & - & 29.9 \\
\hline $\mathrm{R} \mathrm{CrA}$ & IRS 9 & $X-1$ & $0.92(7)$ & 0.489 & 1.36 & 1.5 & - & 52.0 & - & 29.2 \\
\hline
\end{tabular}

${ }^{*}$ In the $0.5-8 \mathrm{keV}$ passband; $L_{\mathrm{x}}$ is absorption corrected and refers to the distance given in Table $2 ;{ }^{a}$ in Obs-ID $3428 ;{ }^{b}$ in Obs-ID 2404.

IRS 9 flared (see Fig. 3), and HD 104237-6 showed a drastic change in count rate by a factor of 8 between the two Chandra exposures. The spectrum of this star refers to the exposure ObsID 3428, where HD 104237-6 was probably caught in a flarelike high state. These flares may partly explain the high observed temperatures. Another possibility is that in the presence of strong absorption the soft spectral component may become unrecognizable. The temperature of $\mathrm{R} \mathrm{CrA}$ is unconstrained, and we give the lower limit in Table 4.

\section{Discussion}

\subsection{The nature of the $X$-ray emitters}

The HAeBe stars observed with Chandra do not represent an unbiased sample because (i) some have been selected for Chandra observations because of their X-ray detections in previous X-ray surveys; and (ii) they cover a vast range of distances and do not form a volume limited sample. X-ray emission for a distance limited, small sample of HAeBe stars has been studied by Skinner et al. (2004), but this group is not complete either and did not contain non-detections. Furthermore, mixing of data obtained with different instruments may introduce uncertainties in the X-ray parameters. Also, note the controversial definition of the HAeBe class. Our sample was selected from published catalogs that contain some dubious cases, e.g. two stars of our sample have spectral type F (ZCMa and HD 152404), and - strictly speaking - are not Herbig Ae/Be objects. For more details on the nature of the individual stars see the Appendix.

A total of 13 out of 17 HAeBe stars are detected. Seven of them have (visual and/or spectroscopic) companions unresolvable with Chandra imaging that might be responsible for the $\mathrm{X}$-ray emission. Five HAeBe stars observed with Chandra have no earlier X-ray data. The detection fraction of the HAeBe stars in this sample is $\sim 76 \%$ if the known or hypothesized companions that remain unresolvable are not taken into consideration, $\sim 53 \%$ if the emission is attributed to known unresolved visual companions, and $\sim 35 \%$ if the emission is attributed to known unresolved visual or spectroscopic companions (cf. Table 1). This result suggests that either a large fraction of HAeBe stars are intrinsic X-ray sources, or a substantial fraction of companions has yet to be discovered.

Similar conclusions on the detection fractions were reached for MS B-type stars observed with Chandra (Stelzer et al. 2006). However, a direct comparison with the detection fractions of HAeBe stars is impeded by the different criteria for the sample selection. The MS B star sample is composed of previously known X-ray emitters for which the presence of faint companions within $1-8^{\prime \prime}$ had been demonstrated before, while for the HAeBe sample no systematic survey for companions exists.

Interestingly, despite the unprecedented 1 " resolution of Chandra, we can establish for only two HAeBe stars (V590 Mon and HD 176386) that previous lower spatial resolution observations erroneously considered them X-ray emitters due to confusion with other objects. (It is assumed here that the nondetections are X-ray dark. This seems justified by the rather stringent upper limits we derive on the X-ray luminosity.) For many of the stars observed with ROSAT and/or ASCA the low spatial resolution data have yielded surprisingly good estimates for the X-ray luminosity, although in other cases $L_{\mathrm{x}}$ was overestimated due to source confusion. This is shown in Fig. 4 which compares the X-ray luminosities derived from Chandra with those from ROSAT and ASCA. Our Chandra imaging has verified that objects located below the diagonal in the diagram were severely contaminated by nearby sources in the ROSAT and ASCA observations.

Strong constraints are placed on the X-ray luminosity of all four undetected HAeBe stars: $\log L_{\mathrm{x}}<28.8 \mathrm{erg} / \mathrm{s}$. This is consistent with the absence of unknown companions of these stars, because virtually all TTS with age of $<10 \mathrm{Myr}$ emit at higher levels; see e.g. Stelzer \& Neuhäuser (2000). In line with this argument, all but one of the four non-detections are not known to be multiple. The exception (HD 147889) is a spectroscopic binary, but composed of two B2-type stars. 


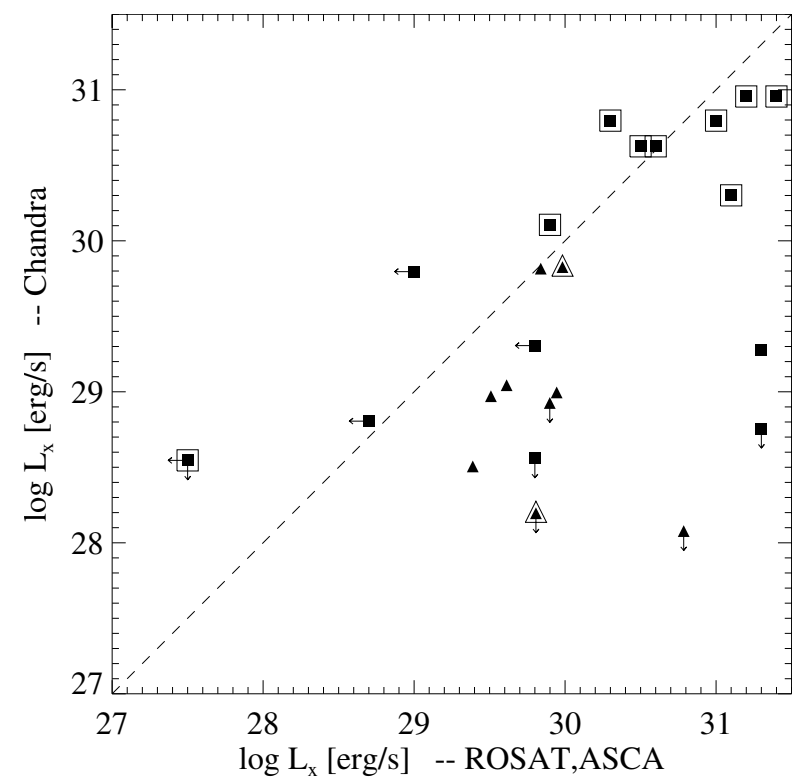

Fig. 4. X-ray luminosities for HAeBe stars (squares) and MS B-type stars (triangles) measured with different instruments. $x$-axis $-L_{\mathrm{x}}$ from ROSAT or ASCA, y-axis $-L_{\mathrm{x}}$ from Chandra. All luminosities refer to the $0.5-8 \mathrm{keV}$ band. ROSAT luminosities are from Zinnecker \& Preibisch (1994) and Hamaguchi et al. (2005) for HAeBe stars and from Berghöfer et al. (1996) for MS B-type stars; ASCA luminosities for HAeBe stars are from Hamaguchi et al. (2005); Chandra luminosities for MS B-type stars are from Stelzer et al. (2006) Binaries that are unresolved with Chandra are surrounded by larger symbols. Three stars (the topmost in terms of Chandra luminosity) appear twice in this diagram because they have both ROSAT and ASCA data.

\subsection{X-ray properties}

To examine the X-ray characteristics of the HAeBe stars and their companions, they are compared to other samples of intermediate- and low-mass stars observed with Chandra: the abovementioned MS B-type stars, and young stars in the Orion Nebula Cluster (ONC). An extensive X-ray study of the ONC was performed in the framework of the Chandra Orion Ultradeep Project (COUP); see ApJ Supplement Special Issue 160.

For the stars with 2-T spectra we computed a mean coronal temperature, defined as the average of the two temperatures weighted with the emission measures. Figure 5 displays the X-ray luminosity versus X-ray temperature for the HAeBe stars (filled squares), the MS B-type stars (filled triangles) and the ONC B/A-type stars (filled circles). Lower mass companions to each of these groups are represented by open symbols, and by dots in the case of the ONC.

The distribution of the HAeBe systems in the $\log L_{\mathrm{x}}-k T$ plot more closely resembles that of young, low-mass stars in the ONC than that of MS B star systems. While the B stars on the MS, as well as most of their companions, are characterized by $k T \sim 0.5 \ldots 1 \mathrm{keV}$, the HAeBe stars show a much larger range with the majority of them at $k T>1 \mathrm{keV}$. For the only exception of a HAeBe star with a low temperature, BD $+30^{\circ} 549$ (already discussed in Sect. 4.3), the fit is ambiguous. The 1T model was chosen as best fit based on statistics. The $1 \mathrm{~T}_{\mathrm{Av}}$ model would place this star at a more typical temperature of $k T \sim 1.3 \mathrm{keV}$ and lower the luminosity to $\log L_{\mathrm{x}} \sim 29.2 \mathrm{erg} / \mathrm{s}$.

The Chandra observations presented here allow for the first time a systematic direct comparison between the X-ray properties of HAeBe stars and their presumably coeval companions.

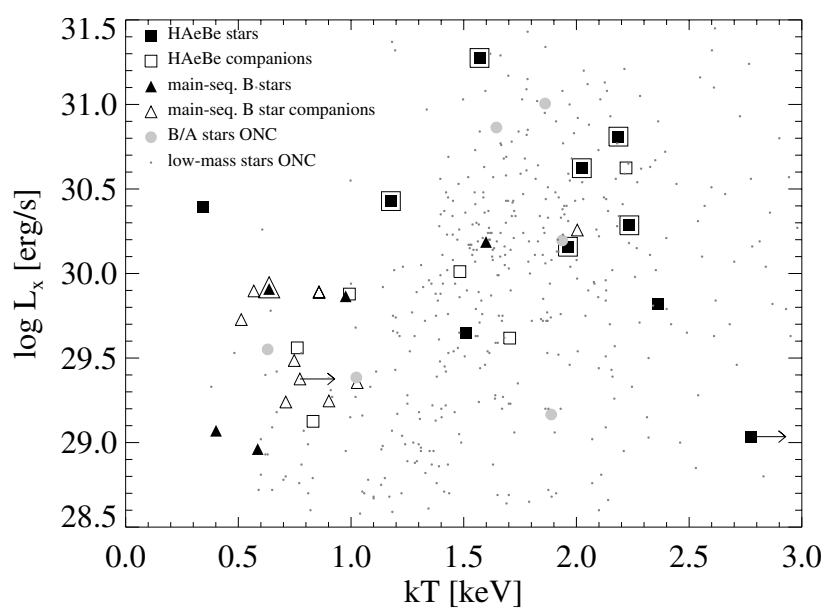

Fig. 5. X-ray temperature and luminosity for $\mathrm{HAeBe}$ stars and their companions compared to main-sequence B stars and companions, and to young stars in Orion; see text Sect. 5.2. Binaries that are unresolved with Chandra are surrounded by larger symbols.

There is some inconclusive indication that the resolved HAeBe companions are softer and less luminous than their primaries. On the other hand, if the unresolved HAeBe companions are also considered X-ray emitters, the distribution is again similar to stars in the low-mass ONC sample.

A comparison of the variability of X-ray sources associated with $\mathrm{HAeBe}$ stars and those ascribed to their resolved companions may, in principle, be employed to discriminate between intrinsic X-rays from HAeBe stars and the companion hypothesis. In the sample of six variable sources, two are apparently single, two are $\mathrm{HAeBe}$ stars with a spectroscopic or unresolved visual companion, one is a resolved companion, and one is an unrelated object (IRS 9). The different lengths of the observations and the different intensities of the sources lead to a wide variety of lightcurves. As far as we can tell, there is no clear distinction between the primaries and the secondaries, but this assertion needs to be corroborated by more extensive X-ray data.

\subsection{Fractional $X$-ray luminosity}

The $L_{\mathrm{x}} / L_{*}$ ratio is examined in Fig. 6 for several samples of intermediate-mass stars in different evolutionary phases. As some HAeBe stars are still surrounded by luminous circumstellar matter, it is important to distinguish between bolometric and stellar luminosity. For stars on the MS $L_{*}$ and $L_{\text {bol }}$ are identical.

The HAeBe stars are compared to the $\sim 1$ Myr old BA stars in the ONC introduced above (see also Stelzer et al. 2005), the $\sim 30$ Myr old BA stars in the Tucanae association (Stelzer \& Neuhäuser 2000), and the MS B stars also introduced above. The latter ones represent on average a slightly hotter and optically brighter sample than the others, because they do not include A-type stars.

A few HAeBe stars have been reported to be very luminous, with $L_{*}>10^{37} \mathrm{erg} / \mathrm{s}$, similar to the range for $\mathrm{O}$ stars. However, we note that the stellar luminosities of some HAeBe stars are highly uncertain (cf. Table 1). In particular, the situation for ZCMa is confusing, with vastly different measurements for $L_{*}$ given in the literature. Throughout this paper we have used $\log \left(L_{*} / L_{\odot}\right)=5.15$ following Acke \& van den Ancker (2004); see Appendix A.6 for more details on the stellar parameters of $\mathrm{ZCMa}$. This object is peculiar in our HAeBe sample because it is highly embedded. 


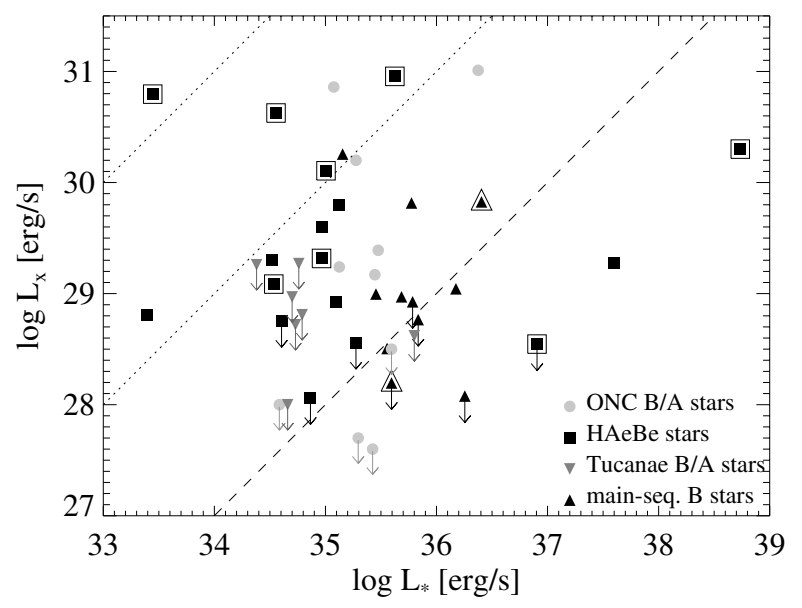

Fig. 6. X-ray versus stellar luminosity for $\mathrm{HAeBe}$ stars and other samples of intermediate-mass stars. The X-ray data is derived from Chandra with the exception of Tucanae, where we use ROSAT All-Sky Survey observations. The dashed line indicates a value of $L_{\mathrm{x}} / L_{\mathrm{bol}}=$ $10^{-7}$, the dotted lines represent $10^{-5}$ and $10^{-3}$, respectively.

The bulk of the HAeBe stars shows $L_{*} \sim 10^{34 \ldots 35} \mathrm{erg} / \mathrm{s}$ and is characterized by a 3 dex spread in X-ray luminosity, with values significantly lower than predicted by the $L_{\mathrm{x}} / L_{*}$ relation of TTS. This was already obvious from the restricted sample presented by Skinner et al. (2004). Two stars, R CrA and V892 Tau, stand out from the sample with untypically low stellar luminosity. The location of these objects below the MS in the HR diagram (Fig. 1) suggests problems in the estimation of $L_{*}$ by the strong circumstellar contribution. Wrong values for $L_{*}$ will also affect the $L_{\mathrm{X}} / L_{*}$ ratio. In fact, V892 Tau has by far the highest fractional X-ray luminosity of the HAeBe stars, $\log \left(L_{\mathrm{X}} / L_{*}\right)>-3$. The $L_{\mathrm{X}} / L_{*}$ ratios given for this star in the previous literature were orders of magnitude lower, but based on the often cited value of $L_{\text {bol }}=38 L_{\odot}$ which is dominated by the contribution from circumstellar material.

The Chandra data constrains the fractional X-ray luminosity of all non-detections of HAeBe stars below $L_{\mathrm{x}} / L_{*} \sim 10^{-6}$. Their range of $L_{\mathrm{x}}$ is similar to that observed in the intermediate-mass stars of the ONC, but the upper limits obtained for the ONC are lower due to the exceptional sensitivity of the COUP. At the age of Tucanae all B- and A-type stars are undetected, and luminosities in excess of $\sim 10^{29} \mathrm{erg} / \mathrm{s}$ can be excluded. The MS B-type sample covers an $L_{\mathrm{x}}$ range similar to the $\mathrm{HAeBe}$ stars (although none of them is found at the high end of the distribution for $\mathrm{HAeBe}$ stars), but there may be a bias towards (strong) X-ray emitters as a result of the selection criteria.

\subsection{X-rays and activity parameters}

In low-mass stars the X-ray luminosity is correlated with indicators for magnetic activity, such as rotation period, $\mathrm{H} \alpha$ emission, and radio continuum emission. The rotation rate of active stars is considered an indicator for the efficiency of the stellar dynamo (e.g. Pallavicini et al. 1981). Therefore, the relation between rotation rates and $\mathrm{X}$-ray luminosities can be used to check the magnetic origin of stellar X-rays. We have used $v \sin i$ as a proxy for rotation, because the rotation periods of the HAeBe stars presented in this work are not known. Photometric monitoring by Herbst \& Shevchenko (1999) searching for periodic variability induced by star spots has led to mostly negative results. Optical variability is present in HAeBe stars, but seems not to be cyclic.

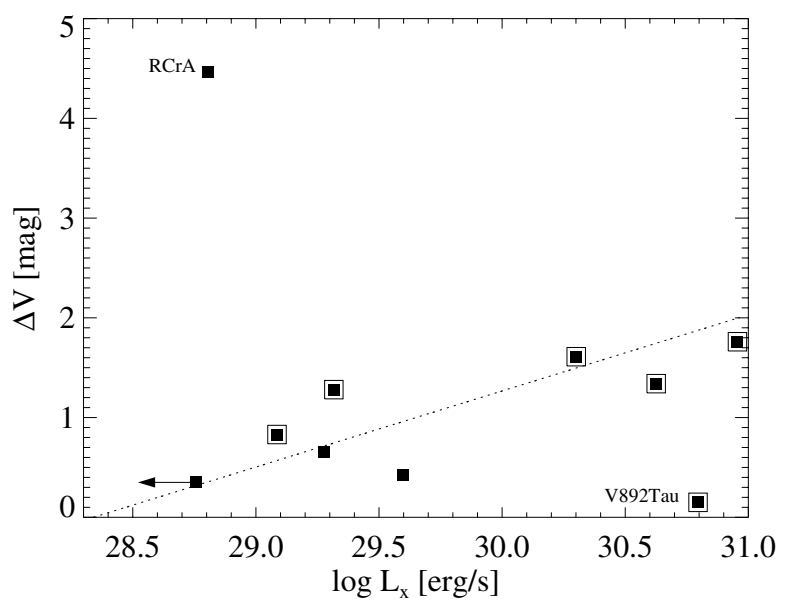

Fig. 7. X-ray luminosity for HAeBe stars versus the amplitude of the $V$ band magnitude from Herbst \& Shevchenko (1999). The dotted line represents a linear regression fit to the data where the two outliers (R CrA and V892 Tau) are excluded.

This kind of variability was termed the UX Ori phenomenon, and it has been ascribed to either variable mass accretion or variable circumstellar obscuration by orbiting dust clumps (Natta et al. 1997; Herbst \& Shevchenko 1999). Therefore, it may not be surprising that we find no evidence for a correlation of $v \sin i$ with the X-ray luminosity. This is also consistent with earlier results from the studies by Zinnecker \& Preibisch (1994) and Hamaguchi et al. (2005). This suggests that - if anything - only a dynamo that is independent of rotation can be at work.

Measurements from optical monitoring are given by Herbst \& Shevchenko (1999) for $10 \mathrm{HAeBe}$ stars from the Chandra sample. With the exception of two stars, there seems to be a trend towards larger variability amplitudes for more X-ray luminous stars (see Fig. 7). The $V$ band amplitude for V892 Tau was determined from only two measurements, while for all other stars the number of observations ranges around several hundred. The other outlier, R CrA, is an extreme case of an UXor object, displaying $V$ band variations in excess of 4 mag. Herbst \& Shevchenko (1999) noted a trend for optically brighter stars to show larger amplitudes. However, the possible connection between $\Delta V$ and $L_{\mathrm{x}}$ shown in Fig. 7 cannot be caused by a relation between $L_{\mathrm{x}}$ and $L_{*}$, because such a correlation is not present in the HAeBe sample (see Fig. 6) where most stars span a small range in stellar luminosity.

The $\mathrm{H} \alpha$ flux of late-type dwarf stars in the field is typically a factor of 3 lower than the X-ray flux (Hawley \& Johns-Krull 2003). Furthermore, all active stars follow a linear relation between the logarithms of their X-ray and radio luminosities (Güdel \& Benz 1993; Güdel et al. 1993). However, this is not the case for the HAeBe stars. They are strongly overluminous in both $\mathrm{H} \alpha$ and radio, and no trend with X-ray emission is seen.

\section{Conclusions}

We have presented the Chandra observations of 17 HAeBe stars, with particular focus on the possible role that late-type companions play in the explanation of the observed X-ray emission. We have compared their X-ray properties to a sample of MS B-type stars and B/A-type stars in the ONC observed with the same instrument, as well as to late-type pre-MS stars.

Based on the present status of their multiplicity, the X-ray emission cannot be explained by known companion stars in a fraction of $\sim 35 \%$ of the sample. To our knowledge no systematic 
studies of binarity in HAeBe stars have been published yet. It is conceivable that all $\mathrm{X}$-rays come from as yet undiscovered latetype companions. Then, the X-ray detection fraction of HAeBe systems in our study would imply a fraction of non-single stars as high as $76 \%$. In practice, however, the Chandra sample may be biased towards stars that had been detected in previous X-ray surveys, and may not be representative for the total population of $\mathrm{HAeBe}$ stars, such that this number must be considered an upper limit to the actual binary fraction.

Alternatively, all or some of the HAeBe stars associated with Chandra sources may produce X-ray emission, either in coronae, star-disk magnetospheres or winds. A comparison of their $\mathrm{X}$-ray characteristics to more evolved intermediate-mass stars is instructive because several of these mechanisms predict declining activity with age. The detection rates for HAeBe stars and MS B-type stars cannot be compared directly because of different sample selection, but there is a clear distinction in terms of $\mathrm{X}$-ray properties. Confirming earlier results obtained with $A S C A$ we find that $\mathrm{HAeBe}$ systems have significantly hotter X-ray plasma than MS B-stars and/or their companions, and somewhat higher X-ray luminosities. These differences might be a sign of fading dynamo action.

Unfortunately, more than one mechanism is consistent with a decrease of activity. One possibility of field generation in fully radiative stars is a shear dynamo that weakens in the course of MS evolution because it is based on the diminuishing rotational energy of the star. On the other hand, the X-ray luminosity and temperature of magnetically active late-type stars also decreases with age (Stelzer \& Neuhäuser 2000; Kastner et al. 2003; Preibisch \& Feigelson 2005). Therefore, cool companions to $\mathrm{HAeBe}$ stars are expected to have hotter coronae than cool companions to MS stars, and all observed X-rays might again be traced back to potential companions. We have shown that the distribution of X-ray temperatures measured for the HAeBe stars is similar to the distribution for TTS in Orion, consistent with this scenario.

Radiatively driven winds are precluded as a cause for the X-rays from HAeBe stars: The terminal wind speeds $\left(\sim 300 \mathrm{~km} \mathrm{~s}^{-1}\right)$ yield a maximum temperature of a few MK at most under the assumption of strong shocks, while the observed $\mathrm{X}$-ray temperatures are higher $(\sim 20 \mathrm{MK})$. For similar reasons, a magnetically confined wind, similar to the model introduced by Babel \& Montmerle (1997a) for Ap stars, is unlikely to apply. In this scenario X-rays are produced in the closed part of the magnetosphere as a consequence of shocks that form upon collision of the winds from the two hemispheres near the equatorial plane. The X-ray luminosities predicted by the model are consistent with the observed values, but the HAeBe winds are too slow to explain the observed high X-ray temperature.

The absence of correlations with activity parameters $(\mathrm{H} \alpha$ emission, radio continuum emission, rotation rate) does not exclude magnetic processes on the HAeBe stars, because the contribution from magnetic activity in the optical and radio may be masked by the stronger effects of winds. Another possibility is $\mathrm{X}$-ray emission related to an accretion shock, similar to the case of cTTS. Magnetospheric accretion has been invoked to explain the shape of $\mathrm{H} \alpha$ line profiles for some HAeBe stars (Muzerolle et al. 2004; Deleuil et al. 2004), but, again, the free-fall velocities $\left(\sim 600 \mathrm{~km} \mathrm{~s}^{-1}\right)$ are inconsistent with the observed high X-ray temperatures.

The incidence of magnetic fields in $\sim 10 \%$ of HAeBe stars is similar to the fraction of magnetic Ap/Bp stars on the MS, and has been taken as evidence that HAeBe stars are the progenitors of magnetic Ap/Bp stars (Wade et al. 2005). If the X-ray emis- sion is related in some way to these fields, one would expect that most of the HAeBe stars of the Chandra sample belong to this small fraction of magnetic stars.

To resolve the mystery of the origin of the X-ray emission from HAeBe stars we strongly encourage the search for magnetic fields on X-ray emitting HAeBe stars, systematic binarity studies of X-ray observed samples to test the companion hypothesis, X-ray surveys on a large and unbiased sample to better constrain detection rates and global X-ray properties, X-ray variability studies of $\mathrm{HAeBe}$ stars recognized to be single to search for magnetic activity, and high spectral resolution X-ray studies of individual objects to probe the density of the emitting plasma.

Acknowledgements. B.S. wishes to thank E. Flaccomio for stimulating discussions. This research has made use of the SIMBAD database, operated at CDS, Strasbourg, France, and the Hipparcos catalogue accessed through the VizieR data base.

\section{References}

Acke, B., \& van den Ancker, M. E. 2004, A\&A, 426, 151

Acke, B., \& Waelkens, C. 2004, A\&A, 427, 1009

Alcalá, J. M., Krautter, J., Schmitt, J. H. M. M., et al. 1995, A\&AS, 114, 109

Alencar, S. H. P., Melo, C. H. F., Dullemond, C. P., et al. 2003, A\&A, 409, 1037

Andersen, L., Lindgren, H., Hazen, M. L., \& Mayor, M. 1989, A\&A, 219, 142

Anderson, I. M., Harju, J., Knee, L. B. G., \& Haikala, L. K. 1997, A\&A, 321, 575

Augereau, J. C., Lagrange, A. M., Mouillet, D., \& Ménard, F. 1999, A\&A, 350, L51

Augereau, J. C., Lagrange, A. M., Mouillet, D., \& Ménard, F. 2001, A\&A, 365, 78

Babel, J., \& Montmerle, T. 1997a, A\&A, 323, 121

Babel, J., \& Montmerle, T. 1997b, ApJ, 485, L29

Bally, J., Feigelson, E. D., \& Reipurth, B. 2003, ApJ, 584, 843

Berghöfer, T. W., Schmitt, J. H. M. M., \& Cassinelli, J. P. 1996, A\&AS, 118, 481

Böhm, T., \& Catala, C. 1995, A\&A, 301, 155

Böhm, T., Catala, C., Balona, L., \& Carter, B. 2004, A\&A, 427, 907

Bouvier, J., \& Corporon, P. 2001, in The Formation of Binary Stars, ed. H. Zinnecker, \& R. D. Mathieu, Proc. IAU Symp., 200, 155

Casey, B. W., Mathieu, R. D., Suntzeff, N. B., Lee, C.-W., \& Cardelli, J. A. 1993, AJ, 105, 2276

Casey, B. W., Mathieu, R. D., Suntzeff, N. B., \& Walter, F. 1995, AJ, 109, 2156 Chauvin, G., Lagrange, A.-M., Beust, H., et al. 2003, A\&A, 406, L51

Corcoran, M., \& Ray, T. P. 1997, A\&A, 321, 189

Corcoran, M., \& Ray, T. P. 1998, A\&A, 331, 147

Corporon, P., \& Lagrange, A.-M. 1999, A\&AS, 136, 429

Damiani, F., Micela, G., Sciortino, S., \& Harnden, F. R. Jr. 1994, ApJ, 436, 807 Damiani, F., Micela, G., \& Sciortino, S. 2006, A\&A, 447, 1041

Deleuil, M., Lecavelier des Etangs, A., Bouret, J.-C., et al. 2004, A\&A, 418, 577 Deleuil, M., Bouret, J. C., Catala, C., et al. 2005, A\&A, 429, 247

de Zeeuw, P. T., Hoogerwerf, R., de Bruijne, J. H. J., Brown, A. G. A., \& Blaauw, A. 1999, AJ, 117, 354

Donati, J.-F., Semel, M., Carter, B. D., et al. 1997, MNRAS, 291, 658 Drew, J. E., Busfield, G., Hoare, M. G., et al. 1997, MNRAS, 286, 538 Ducourant, C., Teixeira, R., Périé, J. P., et al. 2005, A\&A, 438, 769 Eisner, J. A., Lane, B. F., Hillenbrand, L. A., et al. 2004, ApJ, 613, 1049 Elia, D., Strafella, F., Campeggio, L., et al. 2004, ApJ, 601, 1000

Feigelson, E. D., \& Lawson, W. D. 2004, ApJ, 614, 267

Feigelson, E. D., Lawson, W. D., \& Garmire, G. P. 2003, ApJ, 599, 1207

Finkenzeller, U. 1985, A\&A, 151, 340

Flaccomio, E., Micela, G., \& Sciortino, S. 2006, in press

Forbrich, J., Preibisch, Th., \& Menten, K. M. 2006, A\&A, 446, 155

Freeman, P. E., Kashyap, V., Rosner, R., \& Lamb, D. Q. 2002, ApJS, 138, 185

Fuente, A., Martin-Pintado, J., Bachiller, R., Neri, R., \& Palla, F. 1998, A\&A, 334,253

Fukagawa, M., Tamura, M., Itoh, Y., Hayashi, S. S., \& Oasa, Y. 2003, ApJ, 590, L49

Garcia, P. J. V., Thiebaut, E., \& Bacon, R. 1999, A\&A, 346, 892

Gehrels, N. 1986, ApJ, 303, 336

Getman, K. V., Feigelson, E. D., Townsley, L., et al. 2002, ApJ, 575, 354

Giardino, G., Favata, F., Micela, G., \& Reale, F. 2004, A\&A, 413, 669

Grady, C. A., Perez, M. R., \& Thé, P. S. 1993, A\&A, 274, 847

Grady, C. A., Devine, D., Woodgate, B., et al. 2000, ApJ, 544, 895

Grady, C. A., Polomski, E. F., Henning, Th., et al. 2001, AJ, 122, 3396

Grady, C., Woodgate, B., Torres, C. A. O., et al. 2004, ApJ, 608, 809 
Güdel, M., \& Benz, A. O. 1993, ApJ, 405, L63

Güdel, M., Schmitt, J. H. M. M., Bookbinder, J. A., \& Fleming, T. A. 1993, ApJ, 415,236

Habart, E., Testi, L., Natta, A., \& Vanzi, L. 2003, A\&A, 400, 575

Haffner, L. M., \& Meyer, D. M. 1995, ApJ, 453, 450

Hamaguchi, K., Yamauchi, S., \& Koyama, K. 2005, ApJ, 618, 360

Hartmann, L., Kenyon, S. J., Hewett, R., et al. 1989, ApJ, 338, 1001

Hawley, S. L., \& Johns-Krull, C. M. 2003, ApJ, 588, L109

Herbig, G. H. 1960, ApJ, 131, 632

Herbst, W., \& Shevchenko, V. S. 1999, AJ, 118, 1043

Hernandez, J., Calvet, N., Briceño, C., Hartmann, L., \& Berlind, P. 2004, AJ, 127,1682

Hillenbrand, L. A., Strom, S. E., Vrba, F. J., \& Keene, J. 1992, ApJ, 397, 613

Hog, E., Kuzmin, A., Bastian, U., et al. 1998, A\&A, 335, L65

Hu, J. Y., Thé, P. S., \& de Winter, D. 1989, A\&A, 208, 213

Hu, J. Y., Blondel, P. F. C., Catala, C., et al. 1991, A\&A, 248, 150

Hubrig, S., Schöller, M., \& Yudin, R. V. 2004, A\&A, 428, L1

Kardopolov, V. I., Sahanionok, V. V., \& Philipjev, G. K. 1981, Perem. Zvezdy, 21,589

Kastner, J. H., Huenemoerder, D. P., Schulz, N. S., Canizares, C. R., \& Weintraub, D. A. 2002, ApJ, 567, 434

Kastner, J. H., Crigger, L., Rich, M., \& Weintraub, D. A. 2003, ApJ, 585, 878

Kastner, J. H., Franz, G., Grosso, N., et al. 2005, ApJS, 160, 511

Koresko, C. D. 1991, AJ, 102, 2073

Kraft, R. P., Burrows, D. N., \& Nousek, J. A. 1991, ApJ, 374, 344

Leinert, C., Richichi, A., \& Haas, M. 1997, A\&A, 318, 472

Li, W., Evans, N. J., Harvey, P. M., \& Colomé, C. 1994, ApJ, 433, L199

MacFarlane, J. J., \& Cassinelli, J. P. 1989, ApJ, 347, 1090

Millan-Gabet, R., \& Monnier, J. D. 2002, ApJ, 580, L167

Muzerolle, J., D’Alessio, P., Calvet, N., \& Hartmann, L. 2004, ApJ, 617, 406

Natta, A., Grinin, V. P., Mannings, V., \& Ungerechts, H. 1997, ApJ, 491, 885

Palla, F., \& Stahler, S. W. 1999, ApJ, 525, 772

Pallavicini, R., Golub, L., Rosner, R., et al. 1981, ApJ, 248, 279

Pirzkal, N., Spillar, E. J., \& Dyck, H. M. 1997, ApJ, 481, 392

Preibisch, T. 2003, A\&A, 401, 543

Preibisch, T., \& Feigelson, E. D. 2005, ApJS, 160, 390

Prusti, T., Natta, A., \& Palla, F. 1994, A\&A, 292, 593

Ramirez, S. V., Rebull, L., Stauffer, J., et al. 2004, AJ, 127, 2659

Ratzka, T., Köhler, R. \& Leinert, Ch. 2005, A\&A, 437, 611

Reipurth, B., \& Zinnecker, H. 1993, A\&A, 278, 81

Rossiter, R. A. 1943, Publ. Michigan Obs., 8, 133

Rydgren, A. E., \& Vrba, F. J. 1987, PASP, 99, 482

Ryter, Ch. E. 1996, Ap\&SS, 236, 285

Schmitt, J. H. M. M., Golub, L., Harnden, F. R. Jr., et al. 1985, ApJ, 290, 307
Schmitt, J. H. M. M., Robrade, J., Ness, J.-U., Favata, F., \& Stelzer, B. 2005, A\&A, 432, L35

Shatsky, N., \& Tokovinin, A. 2002, A\&A, 382, 92

Shevchenko, V. S., Ezhkova, O. V., Ibrahimov, M. A., van den Ancker, M. E., \& Tjin A Djie, H. R. E. 1999, MNRAS, 310, 210

Siebenmorgen, R., Natta, A., Krügel, E., \& Prusti, T. 1998, A\&A, 339, 134

Siebenmorgen, R., Prusti, T., Natta, A., \& Müller, T. G. 2000, A\&A, 361, 258

Skinner, S. L., \& Yamauchi, S. 1996, ApJ, 471, 987

Skinner, S. L., Brown, A., \& Stewart, R. T. 1993, ApJS, 807, 217

Skinner, S. L., Güdel, M., Audard, M., \& Smith, K. 2004, ApJ, 614, 221

Smith, K. W., Balega, Y. Y., Duschl, W. J., et al. 2005, A\&A, 431, 307

Smith, R. K., Brickhouse, N. S., Liedahl, D. A., \& Raymond, J. C. 2001, ApJ, 556, L91

Snow, T. P., Joseph, C. L., \& Meyer, D. M. 1986, ApJ, 303, 433

Stelzer, B., \& Neuhäuser, R. 2000, A\&A, 361, 581

Stelzer, B., \& Schmitt, J. H. M. M. 2004, A\&A, 418, 687

Stelzer, B., Huélamo, N., Hubrig, S., Zinnecker, H., \& Micela, G. 2003, A\&A, 407, 1067

Stelzer, B., Flaccomio, E., Montmerle, T., et al. 2005, ApJS, 160, 557

Stelzer, B., Huélamo, N., Micela, G., \& Hubrig, S. 2006, A\&A, 452, 1001

Swartz, D. A., Drake, J. J., Elsner, R. F., et al. 2005, ApJ, 628, 811

Teixeira, R., Ducourant, C., Sartori, M. J., et al. 2000, A\&A, 361, 1143

Testi, L., Palla, F., \& Natta, A. 1998, A\&AS, 133, 81

The, P. S., de Winter, D., \& Perez, M. R. 1994, A\&AS, 104, 315

Tout, C. A., \& Pringle, J. E. 1993, MNRAS, 272, 528

Turon, C., Egret, D., Gomez, A., et al. 1993, Hipparcos Input Catalogue, Version 2, Bull. Inf. CDS, 43, 5

van den Ancker, M. E., Thé, P. S., Tjin, A., Djie, H. R. E., et al. 1997, A\&A, 324, L33

van den Ancker, M. E., de Winter, D., \& Tjin, A. Djie H. R. E. 1998, A\&A, 330, 145

van den Ancker, M. E., Blondel, P. F. C., Tjin A Djie H. R. E., et al. 2004, MNRAS, 349, 1516

Vink, J. S., O’Neill, P. M., Els, S. G., \& Draw ,J. E. 2005, A\&A, 438, L21

Wade, G. A., Drouin, D., Bagnulo, S., et al. 2005, A\&A, 442, L31

Waters, L. B. F. M., \& Waelkens, C. 1998, ARA\&A, 36, 233

Weinberger, A. J., Becklin, E. E., Schneider, G., et al. 1999, ApJ, 525, L53

Weinberger, A. J., Rich, R. M., Becklin, E. E., Zuckerman, B., \& Matthews, K. 2000, ApJ, 544, 937

Weisskopf, M. C., Brinkman, B., Canizares, C., et al. 2002, PASP, 114, 1

Wilms, J., Allen, A., \& McCray, R. 2000, ApJ, 542, 914

Wolk, S. J., Harnden, F. R., Jr., Flaccomio, E., et al. 2005, ApJS, 160, 423

Yorke, H., \& Sonnhalter, C. 2002, ApJ, 569, 846

Zinnecker, H., \& Preibisch, Th. 1994, A\&A, 292, 152 
B. Stelzer et al.: On the origin of the X-ray emission from HAeBe stars, Online Material $p 1$

\section{Online Material}


B. Stelzer et al.: On the origin of the X-ray emission from HAeBe stars, Online Material $p 2$

Table 2. Target list of HAeBe stars observed with Chandra: position, distance, and spectral type of primaries; separation, position angle, and identifier of companion candidates; observation ID, instrument, exposure time, and reference to previous publication for Chandra observations.

\begin{tabular}{|c|c|c|c|c|c|c|c|c|c|c|c|c|}
\hline \multirow{3}{*}{ Designation } & \multicolumn{3}{|c|}{ HAeBe primaries } & \multirow{3}{*}{$\mathrm{SpT}^{b}$} & \multicolumn{4}{|c|}{ Companions } & \multicolumn{4}{|c|}{ ACIS observations } \\
\hline & \multicolumn{2}{|c|}{ Position $^{a}$} & \multirow{2}{*}{$\begin{array}{r}\text { Dist }^{a} \\
{[\mathrm{pc}]}\end{array}$} & & \multirow{2}{*}{$\begin{array}{c}\mathrm{Sep}^{c} \\
{\left[{ }^{\prime \prime}\right]}\end{array}$} & \multirow{2}{*}{$\begin{array}{l}\mathrm{PA}^{c} \\
{\left[{ }^{\circ}\right]} \\
\end{array}$} & \multirow{2}{*}{ Component } & \multirow{2}{*}{ Ref. } & \multirow{2}{*}{ ObsID } & \multirow{2}{*}{ Instr } & \multirow{2}{*}{$\begin{array}{r}\text { Expo } \\
{[\mathrm{s}]}\end{array}$} & \multirow{2}{*}{ Ref. } \\
\hline & $\alpha_{2000}$ & $\delta_{2000}$ & & & & & & & & & & \\
\hline $\mathrm{BD}+30^{\circ} 549$ & 03:29:19.78 & $+31: 24: 57.0$ & 390 & B8 V pe & - & - & & & 0642 & I & 43351.2 & (17) \\
\hline V892 Tau & 04:18:40.61 & $+28: 19: 15.5^{*}$ & $162^{*}$ & A6e & $\begin{array}{l}4.10 \\
0.05\end{array}$ & $\begin{array}{r}23.4 \\
50\end{array}$ & $\begin{array}{l}\mathrm{B} \\
\mathrm{C}\end{array}$ & $\begin{array}{l}(1) \\
(2)\end{array}$ & 3364 & $S$ & 17734.2 & (18) \\
\hline V380 Ori & $05: 36: 25.43$ & $-06: 42: 57.7$ & $460^{*}$ & B8/A1 e & $\begin{array}{l}0.15 \\
\mathrm{SE}\end{array}$ & $\mathrm{B}^{204.2}$ & B & $\begin{array}{l}\text { (1) } \\
(3)\end{array}$ & 0021 & $S$ & 19510 & \\
\hline HD 147889 & $06: 25: 24.32$ & $-24: 27: 56.6$ & 136 & $\mathrm{~B} 2 \mathrm{~V}$ & $\mathrm{SP}$ & & & (4) & 0618 & $\mathrm{I}$ & 5014.1 & \\
\hline V590 Mon & $06: 40: 44.64$ & $+09: 48: 02.2^{*}$ & $800^{*}$ & B8 ep+sh & - & - & & & 2550 & I & 48138 & (19) \\
\hline $\mathrm{ZCMa}$ & 07:03:43.16 & $-11: 33: 06.2$ & $1050^{*}$ & F6 III e & 0.11 & 129 & B & (5) & 3751 & $S$ & & \\
\hline HD 97300 & 11:09:50.02 & $-76: 36: 47.7$ & 188 & B9 V & - & - & & & 1867 & I & 66292.1 & (20) \\
\hline HD 100546 & $11: 33: 25.44$ & $-70: 11: 41.2$ & 103 & B9 Vne & $\begin{array}{l}4.54 \\
5.22 \\
5.91 \\
5.55\end{array}$ & $\begin{array}{r}196.5 \\
155.1 \\
26.4 \\
322.6\end{array}$ & & $\begin{array}{l}(6) \\
(6) \\
(6) \\
(6)\end{array}$ & 2403,3427 & $\mathrm{I}$ & 2622,2628 & (21) \\
\hline HD 104237 & 12:00:05.08 & $-78: 11: 34.6$ & 116 & $\mathrm{~A} 0 \mathrm{Vpe}$ & $\begin{array}{r}5.28 \\
1.37 \\
10.72 \\
14.88 \\
\text { SE }\end{array}$ & $\begin{array}{r}310.1 \\
254.6 \\
117 \\
121 \\
\text { B }\end{array}$ & $\begin{array}{l}1 \\
2 \\
5 \\
6\end{array}$ & $\begin{array}{c}(7) \\
(7) \\
\left(7^{*}\right) \\
\left(7^{*}\right) \\
(8)\end{array}$ & 2404,3428 & I & 2904,2828 & (21) \\
\hline HD 141569 & $15: 49: 57.75$ & $-03: 55: 16.4$ & 99 & B9.5 Ve & $\begin{array}{l}7.57 \\
8.93\end{array}$ & $\begin{array}{l}311.5 \\
310.0\end{array}$ & $\begin{array}{l}\text { B } \\
\text { C }\end{array}$ & $\begin{array}{l}(9) \\
(9)\end{array}$ & 0981 & $\mathrm{I}$ & 2916 & (21) \\
\hline HD 150193 & $16: 40: 17.92$ & $-23: 53: 45.2$ & 150 & $\mathrm{~A} 1 \mathrm{Ve}$ & $\begin{array}{l}1.10 \\
\mathrm{SE}\end{array}$ & $\mathrm{B}^{225}$ & B & $\begin{array}{l}\text { (10) } \\
(3)\end{array}$ & 0982 & I & 2919 & (21) \\
\hline HD 152404 & $16: 54: 44.85$ & $-36: 53: 18.6$ & 145 & F5 V & $\mathrm{SE}$ & & & (11) & 0983 & I & 3112.1 & (21) \\
\hline HD 163296 & $17: 56: 21.29$ & $-21: 57: 21.9$ & 122 & $\mathrm{~A} 1 \mathrm{Ve}$ & - & - & & (12) & 3733 & $\mathrm{~S}$ & 20000 & (22) \\
\hline MWC 297 & $18: 27: 39.65$ & $-03: 49: 52.0^{*}$ & $250^{*}$ & $\mathrm{O} 9 \mathrm{e}$ & 3.39 & 313 & B & (13) & 1883 & I & 40000 & $(15,23)$ \\
\hline HD 176386 & 19:01:38.93 & $-36: 53: 26.5$ & 136 & B9 IV & 4.1 & 138 & B & (14) & 0019 & I & 37330 & \\
\hline TY CrA & 19:01:40.83 & $-36: 52: 33.9^{*}$ & $136 ?$ & B9e & $\begin{array}{l}0.29 \\
\mathrm{SE}\end{array}$ & $\mathrm{B}^{188.5}$ & B & $\begin{array}{l}\text { (15) } \\
(16)\end{array}$ & 0019 & I & 37330 & \\
\hline $\mathrm{RCrA}$ & 19:01:53.65 & $-36: 57: 07.6$ & $130^{*}$ & A1 e-F7 e var & - & - & & & 0019 & I & 37330 & (24) \\
\hline
\end{tabular}

* For two companions to HD 104237 the position angle from the literature does not coincide with the published IR image. The position angles cited in this table and used throughout this paper are corrected values (Grady, priv. comm.). ${ }^{a}$ Hipparcos position and distance; exceptions are data marked with * where positions are from Ducourant et al. (2005), Hog et al. (1998), and The et al. (1994), and distances from Hillenbrand et al. (1992), Shevchenko et al. (1999), van den Ancker et al. (1998), and Drew et al. (1997); ${ }^{b}$ spectral types adopted from The et al. (1994), van den Ancker et al. (1997), or van den Ancker et al. (1998); " "SB" stands for spectroscopic companions. References: (1) Leinert et al. (1997); (2) Smith et al. (2005); (3) Corporon \& Lagrange (1999); (4) Haffner \& Meyer (1995); (5) Millan-Gabet \& Monnier (2002); (6) Shatsky \& Tokovinin (2002); (7) Grady et al. (2004); (8) Böhm \& Catala (2004); (9) Weinberger et al. (1999); (10) Fukagawa et al. (2003); (11) Andersen et al. (1989); (12) Grady et al. (2000); (13) Vink et al. (2005); (14) Turon et al. (1993); (15) Chauvin et al. (2003); (16) Casey et al. (1995); (17) Getman et al. (2002); (18) Giardino et al. (2004); (19) Ramirez et al. (2004); (20) Feigelson et al. (2004); (21) Feigelson et al. (2003); (22) Swartz et al. (2005); (23) Damiani et al. (2006); (24) Forbrich et al. (2006). 


\section{Appendix A: Individual systems}

\section{A.1. $B D+30^{\circ} 549$}

The B9 star BD $+30^{\circ} 549$ is the optically brightest star of the NGC 1333 star-forming region.

Undetected with both ROSAT and ASCA (Hamaguchi et al. 2005), the first X-ray detection of this HAeBe star was presented by Getman et al. (2002) based on the Chandra observation; source 83 . Our re-analysis of the Chandra image confirms that the X-ray luminosity amounts to $\log L_{\mathrm{x}}=29.3 \mathrm{erg} / \mathrm{s}$, well below the detection limits of the previous X-ray observations. A weak, non-variable source at the position of $\mathrm{BD}+30^{\circ} 549$ was also seen with XMM-Newton (Preibisch 2003). The spectral parameters we derive are in agreement with previous determinations by Getman et al. (2002) and Preibisch (2003).

\section{A.2. V892 Tau}

V892 Tau is a HAeBe star in the Taurus cloud complex. Next to the primary of spectral type B9...A6 the system includes a $\mathrm{T}$ Tauri type companion at a separation of $\sim 4^{\prime \prime}$ (Leinert et al. 1997). Smith et al. (2005) discovered a further faint companion with estimated mass of $1.5-2 M_{\odot}$ at only 50 mas from the HAeBe star.

V892 Tau was observed by both XMM-Newton and Chandra, however only Chandra was able to resolve the wider pair. Two $\mathrm{X}$-ray sources are seen. The brighter one was identified by Giardino et al. (2004) with the HAeBe star. This observation was particularly intriguing because of an X-ray flare in the Chandra data (see Fig. 3). After the discovery of the further sub-arcsec companion, which remains unresolved in the X-ray data, the association of the flare with the HAeBe star must now be questioned. This companion possibly features a shallow convection zone, and may have $L_{*}$ almost as high as the HAeBe primary. Therefore, it would yield a "reasonable" value for $\log \left(L_{\mathrm{x}} / L_{*}\right)$ if responsible for the X-ray emission.

The spectrum we extract in our re-analysis of the Chandra data indicates a $\sim 2 \mathrm{keV}$ plasma for the brighter X-ray component (identified with the system of HAeBe star plus 50 mas companion), absorbed by a hydrogen column density of $\sim 1.4 \times$ $10^{22} \mathrm{~cm}^{-2}$, and a luminosity of $10^{30.7} \mathrm{erg} / \mathrm{s}$, in rough accordance with the values quoted by Giardino et al. (2004). Note that we used a slightly different distance $(162 \mathrm{pc}$ versus $140 \mathrm{pc})$. The emission measure of the companion, V892Tau NE, is smaller by a factor of 10 , has a soft component very similar in shape to that of V892Tau A, but the hard component is weaker.

\section{A.3. V380 Ori}

V380 Ori is a visual binary of separation 0.154 " (Leinert et al. 1997). Probably both components are HAeBe stars. In addition Corporon \& Lagrange (1999) detected the Li line in the spectrum of V380 Ori A, indicating the existence of a further latetype companion. Testi et al. (1998) found no evidence for additional faint IR sources surrounding the star, and argued that the detection of such companions might be impeded by the diffuse nebulosity seen in the $J$ and $K$ band images.

V380 Ori was detected as a strong X-ray source during a ROSAT pointing (Zinnecker \& Preibisch 1994). An X-ray spectrum obtained with $A S C A$ showed rather high plasma temperature $(\sim 3.2 \mathrm{keV}$; Hamaguchi et al. 2005). V380 Ori is in the field of a $20 \mathrm{ks}$ long archived Chandra pointing whose prime target was the Herbig-Haro object HH 1. Due to the small binary separation even the superior angular resolution of Chandra is not sufficient to resolve the system, such that the origin of the single X-ray source remains obscure. The Chandra spectrum can be described reasonably well with a 1-T model of $k T \sim 1.6 \mathrm{keV}$, and column density within a factor of two of the value that corresponds to the visual extinction. The X-ray luminosity $\left(\log L_{\mathrm{x}}=31.3 \mathrm{erg} / \mathrm{s}\right)$ is at the upper end of the range observed in this sample. Previous X-ray satellites have measured similarly high X-ray luminosity.

A longitudinal magnetic field of $0.5 \mathrm{kG}$ was detected in spectropolarimetric measurements (Wade et al. 2005).

\section{A.4. $H D 147889$}

HD 147889 is located in the $\rho$ Oph cloud complex. It is a known double-lined spectroscopic binary composed of two nearly equal B2-type stars (Haffner \& Meyer 1995), but has no known visual companion. In particular, a speckle search yielded a negative result (Ratzka et al. 2005). It is generally assumed that HD 147889 lies behind denser material than other lines of sight within $\rho$ Oph (see Snow et al. 1986, and references therein).

HD 147889 was in the sample of HAeBe stars studied by Hamaguchi et al. (2005), but no X-ray emission was reported. The star is also undetected with Chandra down to a detection limit of $\log L_{\mathrm{x}}<28.6 \mathrm{erg} / \mathrm{s}$, corresponding to one of the strongest constraints for the fractional X-ray luminosities of HAeBe stars measured so far $\log \left(L_{\mathrm{x}} / L_{*}\right)<-8.4$.

\section{A.5. V590Mon}

V590 Mon (=LkH $\alpha 25)$ is an enigmatic object in the NGC 2264 cluster: in the HR diagram it is located below the MS but it shows signs of pre-MS status, most notably an extreme IR excess (Rydgren \& Vrba 1987), leading to the speculation that this object suffers significant extinction arising from a disk viewed edge-on. From the absence of both $\mathrm{mm}$ - or cm-continuum emission Fuente et al. (1998) concluded that this star is located in a cavity of the molecular cloud. The speckle search for binaries among HAeBe stars by Leinert et al. (1997) was negative for this star.

The NGC 2264 cluster was observed for $50 \mathrm{ks}$ with Chandra (Ramirez et al. 2004). We find an upper limit of $\log L_{\mathrm{x}}<$ $28.8 \mathrm{erg} / \mathrm{s}$ for V590Mon, consistent with the value cited by Ramirez et al. (2004). Its non-detection is in accordance with earlier (less sensitive) X-ray observations. An X-ray source is identified $\sim 10.6$ North of V590 Mon in the small portion of the image we analysed, corresponding to source No.103 of Ramirez et al. (2004), a K7 star.

\section{A.6. ZCMa}

The perception of ZCMa, originally classified as $\mathrm{HAeBe}$ star (Herbig et al. 1960), has dramatically changed as more observational details were revealed.

$\mathrm{ZCMa}$ is a young F-type star with FU Ori like characteristics, a mass of $\sim 3 M_{\odot}$, bolometric luminosity of $28 L_{\odot}$, and an age of $\sim 3 \times 10^{5} \mathrm{yrs}$, just below the birthline in the HR diagram (Hartmann et al. 1989). A powerful IR source is found at only 0.1" (Koresko et al. 1991). Assuming that this object is coeval with the FU Or star, van den Ancker et al. (2004) showed that it can be modeled as a B0 type star on the birthline with a luminosity of $\log \left(L_{*} / L_{\odot}\right)>5$ and a mass of $38 M_{\odot}$. Therefore, this is the actual primary of the system. In traditional models (see 
Fig. 1) the star is placed above the birthline. However, calculations that include the effect of dust on the evolution of a massive star raise the birthline (Yorke \& Sonnhalter 2002), such that the high observed luminosity can be accomodated.

The system is characterized by occasional outbursts in optical brightness. Although the optical light is usually dominated by the FU Or star, the spectrum during outburst is dominated by the IR companion, which drives a jet and bipolar outflow (Garcia et al. 1999). The origin of the outbursts is not well understood. They may be related to changes in the magnetic field configuration that entail changes in the outflow (van den Ancker et al. 2004).

With Chandra we find two X-ray sources in the vicinity of $\mathrm{ZCMa}$, one on the position of the unresolved binary and another unidentified and very faint one $\sim 8.6^{\prime \prime}$ to the south. If the stellar luminosity of ZCMa is indeed as high as assumed throughout this paper $\left(\log \left(L_{*} / L_{\odot}\right)=5.15\right.$; Acke \& van den Ancker 2004), the upper limit for $\log \left(L_{\mathrm{X}} / L_{*}\right)(\sim-8.4)$ is very low despite the fact that the X-ray luminosity is among the highest of the HAeBe stars.

\section{A.7. HD 97300}

HD 97300 is located in the Cha I star forming region. Mid-IR images show that HD 97300 is surrounded by a dust ring possibly created by interaction of the star with circumstellar matter, and a secondary peak of the ISOCAM image suggests the presence of an IR companion $\sim 3^{\prime \prime}$ north of the primary which is detected only above $10 \mu \mathrm{m}$ (Siebenmorgen et al. 1998).

HD 97300 was detected with ROSAT (see Hamaguchi et al. 2005) at $\log L_{\mathrm{x}}=29.0 \mathrm{erg} / \mathrm{s}$. Feigelson et al. (2004) discussed the Chandra image of Cha I North, where HD 97300 was identified as their source 44. In our restricted Chandra image the only $\mathrm{X}$-ray source, brighter by $\sim 1$ dex than deduced from the ROSAT data, is clearly associated with HD 97300 itself. The decaying phase of a flare is seen at the beginning of the observation, but the flare is not strong enough to make a significant difference between the quiescent and the average count rate. There is no evidence for X-ray emission from the IR companion.

\section{A.8. $H D 100546$}

HD 100546 is a B9.5 Ve HAeBe star (Hu et al. 1989), and with an age of $>10 \mathrm{Myr}$ one of the most evolved objects of this class (van den Ancker et al. 1997). Evidence for its rather high age comes from the $\lambda$ Boo like abundance anomalies that hint at gas-dust separation and ensuing metal depletion of the accreted material, thought to take place in the late pre-MS phase (Acke \& Waelkens 2004b). Augereau et al. (2001) and Shatsky \& Tokovinin (2002) reported the detection of four faint IR objects within $\sim 10^{\prime \prime}$ of HD 100546 (see also Grady et al. 2001, for a cartoon on the environment of HD 100546). Based on the near-IR photometry all four companions were classified as "optical", i.e. no true physical companions. This conclusion is in agreement with the non-detection of these objects with Chandra. Their limiting X-ray luminosity is $\log L_{\mathrm{x}}=27.9 \mathrm{erg} / \mathrm{s}$ if assumed to be at the same distance as HD 100546.

HD 100546 is surrounded by a circumstellar dust disk with cometary-like chemical composition. The Chandra observation of HD 100546 was discussed by F03. We found no previous reports on X-ray emission from this star. In our re-analysis of the Chandra data we detect it at $\log L_{\mathrm{x}}=28.9 \mathrm{erg} / \mathrm{s}$, discrepant with the value given by F03 by 0.4 dex. The plasma temperature we assume in deriving the $\mathrm{X}$-ray luminosity is smaller ( $1 \mathrm{keV}$ versus $2.5 \mathrm{keV}$ of F03). As the absorption is similar (fixed to $A_{\mathrm{V}}=0.06$ in our case and neglected by F03) the difference in $L_{\mathrm{x}}$ remains unexplained.

\section{A.9. $H D 104237$}

HD 104237 is the optically brightest Herbig star. It is located in the Chamaeleon star forming complex, but outside the boundaries of the molecular clouds. Although showing signatures typical of HAeBe stars (emission lines, IR excess from a dust shell) HD 104237 is not surrounded by a reflection nebula (Hu et al. 1989) that would indicate association with a star forming cloud.

Furthermore, from an analysis of radial velocities and the presence of lithium absorption in the spectrum of HD 104237 Böhm \& Catala (2004) concluded that it is a spectroscopic binary. An IUE observation of HD 104237 has shown evidence for hot plasma (Hu et al. 1991). The star is listed as a RASS X-ray source by Alcalá et al. (1995), and was also detected in a dedicated observation by ASCA (Skinner \& Yamauchi 1996; Hamaguchi et al. 2005). Feigelson et al. (2003) resolved the $\mathrm{X}$-ray emission from this object with two Chandra snapshots into five individual sources that likely form a young stellar group. No close visual companions were known prior to this study, but optical/IR identifications for the new X-ray sources have meanwhile been provided (Grady et al. 2004).

In our reanalysis of the Chandra data we find only three sources, identified as HD 104237 A, HD 104237-5, and HD 104237-6. The brightest one is the HAeBe star. As discussed in the text, HD 104237-2 is probably missed because of its small separation from HD 104237 A.

\section{A.10. $H D 141569$}

The evolutionary state of HD 141569 is ambiguous. It exhibits characteristics of both HAeBe stars (emission lines, association with a reflection nebula) and MS stars (low ratio of infrared to stellar luminosity). van den Ancker et al. (1998) conclude that HD 141569 may be a transition object, consistent with its position in the HR diagram very close to the ZAMS. The $4^{\prime \prime}$ wide disk was imaged in scattered light (Weinberger et al. 1999; Augereau et al. 1999). These images were sensitive to the detection of planets down to $3 M_{\text {Jup }}$, but did not detect any such object, thus the existence of very close-in companions is unlikely. A search for spectroscopic binaries among HAeBe stars also provided a negative result for HD 141569 (Corporon \& Lagrange 1999).

Two potential companions within $<10^{\prime \prime}$ of HD 141569 have first been identified by Rossiter (1943). Recently the position and IR photometry of these objects were discussed by Weinberger et al. (2000). These have shown that the system forms a physical triple; astrometry confirms that both companions are bound, and the presence of lithium absorption in their spectrum establishes their pre-MS nature.

The Chandra observation of HD 141569 was presented by Feigelson et al. (2003), who reported a detection for HD 141569 A but no X-ray emission from the two visual companions. These results were picked up by Skinner et al. (2004). In our re-analysis of the same data we find another interpretation for the assignment of counterparts to the X-ray sources: As shown in Fig. 2 the two X-ray sources can clarly be identified with the two companions, while the HAeBe star is X-ray dark down to the detection limit, with $L_{\mathrm{x}}<10^{28} \mathrm{erg} / \mathrm{s}$. 


\section{A.11. HD 150193}

This HAeBe star, also known as MWC 863, is located near the $\rho$ Oph cloud, but isolated. A visual companion was first discovered by Reipurth \& Zinnecker (1993). This object shows T Tauri star characteristics (Bouvier \& Corporon 2001) and is coeval with the primary (Fukagawa et al. 2003). Later HD 150193 A turned out to be a spectroscopic binary with an additional cool companion identified by its $\mathrm{Li}$ absorption (Corporon \& Lagrange 1999). The primary in this system is surrounded by a circumstellar disk. No disk was detected around the visual companion.

Based on the Chandra image Feigelson et al. (2003) considered the companion undetected, and claimed a new companion " $C$ " identified by means of its X-ray emission. In our reanalysis of the Chandra data we detect a single, elongated X-ray source midway between the HAeBe star and its known companion located south-west of the primary. Visually we identify two marginally separated sources, one of which is the "companion C" of F03. However, our cross-correlation with the optical/IR positions suggests that this weak X-ray source to the NE of the brighter source should be identified with the HAeBe star, while the brighter source itself coincides with the T Tauri companion (see our Fig. 2; and Skinner et al. 2004).

\section{A.12. HD 152404}

Strictly speaking HD 152404 is not a HAeBe star, because its spectral type is F5. The Upper Sco member (de Zeeuw et al. 1999 ) is an eccentric double-lined spectroscopic binary system composed of two nearly equal mass stars (Andersen et al. 1989). The profiles of the Balmer emission lines provide evidence for both accretion and outflow, and the SED can be modeled by a circumbinary disk with a large inner gap (Alencar et al. 2003)

At the position of HD 152404 the Chandra image presents one faint X-ray source (see Fig. 2), also reported by Feigelson et al. (2003).

\section{A.13. $H D 163296$}

HD 163296 is a well-studied isolated nearby HAeBe star of intermediate age. It has a dust disk, and is associated with a series of $\mathrm{HH}$ objects (Grady et al. 2000). The rich emission line spectrum of HD 163926 was studied by Deleuil et al. (2005), revealing P Cyg features indicative of a wind and evidence for a chromosphere (heated region). The line profiles of highly ionized species can be explained by magnetospheric accretion or by magnetospherically confined wind shocks.

The Chandra observation of HD 163926 was discussed by Swartz et al. (2005), who derived spectral parameters compatible with our results. They speculated that this star may represent a case of X-ray emission from an accretion shock because of the untypically low temperature. Another possibility is that the soft $\mathrm{X}$-rays arise from shocks at the interface of the accretion disk with the optical jet, as in the cases of L1551 IRS 5 (Bally et al. 2003) and the Beehive Proplyd (Kastner et al. 2005).

\section{A.14. MWC 297}

MWC 297 is one of the hottest and most absorbed HAeBe stars (spectral type O9e; $A_{\mathrm{V}}=8 \mathrm{mag}$ ). Drew et al. (1997) studied this object in detail, and provided an updated distance estimate. Recently a circumstellar disk was resolved (Eisner et al. 2004).
Vink et al. (2005) have identified a faint object at 3.4" from MWC 297 in coronographic adaptive optics $H$-band images.

In light of the discovery of a close companion, the report of a large X-ray flare from MWC 297 in low-spatial resolution ASCA data by Hamaguchi et al. (2005) must be questioned. If it truely occurred on MWC 297 this would be one of only two X-ray flares ascribed to HAeBe stars so far. However, Damiani et al. (2006) have shown from their analysis of Chandra data that there are no less than $9 \mathrm{X}$-ray sources in the ASCA point-spread function, and MWC 297 is not even the brightest of these. We have analysed the same Chandra data and found two sources in the small $(100 \times 100$ pixels $)$ image. These two X-ray sources coincide with MWC 297 and its adaptive optics companion. Both sources are too faint for spectral analysis. But the X-ray luminosity for the MWC 297 source $\left(\log L_{\mathrm{x}}=29.3 \mathrm{erg} / \mathrm{s}\right)$ is magnitudes below the estimate from ASCA $\left(\log L_{\mathrm{x}} \sim 31 \ldots 32 \mathrm{erg} / \mathrm{s}\right)$.

\section{A.15. $H D 176386$}

HD 176386 shows far-IR excess detected with IRAS indicating emission from a dust shell. Its mid-IR spectrum is dominated by extended emission (Prusti et al. 1994) and shows two peaks, the stronger of which is associated with the position of the star (Siebenmorgen et al. 2000). Although close to the ZAMS in the HR diagram, the presence of C IV absorption lines in IUE spectra of HD 176386 with wings extending out to velocities of several hundreds of $\mathrm{km}$ was interpreted as evidence for accretion of circumstellar gas onto the star (Grady et al. 1993).

HD 176386 shares proper motion with TY CrA, from which it is separated by $\sim 55^{\prime \prime}$, and the two stars may be physically associated with each other (Teixeira et al. 2000). From the Hipparcos data base (Turon et al. 1993) we find that HD 176386 is a binary with $4.1^{\prime \prime}$ separation. The secondary is $\sim 5 \mathrm{mag}$ fainter.

Hamaguchi et al. (2005) reported an X-ray detection of HD 176386 with $A S C A$, but the object was only marginally resolved from TY CrA. The Chandra data presented here not only confirms the detection, but demonstrates also that the X-ray source is associated with the companion. The HAeBe star itself is X-ray dark down to our detection limit of $\log L_{\mathrm{x}}<28.6 \mathrm{erg} / \mathrm{s}$.

\section{A.16. TYCrA}

TY CrA forms a multiple system. The central eclipsing binary (Kardopolov et al. 1981), a spectroscopic companion with $M \sin ^{3} i=2.4 \pm 0.5 M_{\odot}$ (Casey et al. 1995), and a visual $\sim$ M4-type companion at $\sim 0.3^{\prime \prime}$ separation (Chauvin et al. 2003). The central binary system is composed of the Herbig Be primary and a $\sim 1.6 M_{\odot}$ secondary star in a circular orbit with period of $\sim 2.89 \mathrm{~d}$ (Casey et al. 1993). The lack of near-IR emission and the small extinction of 3 mag does not favor the presence of an optically thick disk (Casey et al. 1993).

X-ray emission from TY CrA has been observed with ROSAT, Einstein and ASCA (Zinnecker \& Preibisch 1994; Damiani et al. 1994; Hamaguchi et al. 2005). The X-ray properties of all these observations are in good agreement with our Chandra measurements. It is one of the X-ray brightest HAeBe stars $\left(\log L_{\mathrm{x}} \sim 30.9 \mathrm{erg} / \mathrm{s}\right)$. TY CrA has shown a flare during the Chandra observation, during which it has roughly doubled its count rate (Fig. 3). The individual components of the quadruple cannot be resolved, such that the only X-ray source may in principle be composed of contributions from all four stars. 


\section{A.17. RCrA}

R CrA is a presumably single HAeBe star (Leinert et al. 1997) surrounded by a reflection nebula that scatters its variable light. The molecular cloud surrounding R CrA contains a number of embedded IR objects, that might be the driving source for some nearby HH outflows (Anderson et al. 1997).

While R CrA remained undetected with ROSAT (Zinnecker $\&$ Preibisch 1994) and ASCA (Hamaguchi et al. 2005), a recent X-ray detection with Chandra was discussed by Skinner et al. (2004) and Forbrich et al. (2006). In our re-analysis of the Chandra image we derive a luminosity of $\log L_{\mathrm{x}}=28.8 \mathrm{erg} / \mathrm{s}$, lower by a factor of two from the value cited by Skinner et al. (2004). The difference can partly be attributed to the different distance estimate used. The X-ray spectrum is dominated by hard emission, and is similar to a nearby class I protostar IRS 9. 